DAMTP-97-101

September, 1997

\title{
ON FUSION ALGEBRAS AND MODULAR MATRICES*
}

\author{
T. Gannon and M.A. Walton ${ }^{\dagger}$ \\ Mathematics Department, York University \\ North York, Ontario, Canada M3J 1P3 \\ tgannon@mathstat.yorku.ca \\ Department of Applied Mathematics and Theoretical Physics \\ University of Cambridge \\ Silver Street, Cambridge CB3 9EW, U.K. \\ m.a.walton@damtp.cam.ac.uk
}

\begin{abstract}
We consider the fusion algebras arising in e.g. Wess-Zumino-Witten conformal field theories, affine Kac-Moody algebras at positive integer level, and quantum groups at roots of unity. Using properties of the modular matrix $S$, we find small sets of primary fields (equivalently, sets of highest weights) which can be identified with the variables of a polynomial realization of the $A_{r}$ fusion algebra at level $k$. We prove that for many choices of rank $r$ and level $k$, the number of these variables is the minimum possible, and we conjecture that it is in fact minimal for most $r$ and $k$. We also find new, systematic sources of zeros in the modular matrix $S$. In addition, we obtain a formula relating the entries of $S$ at fixed points, to entries of $S$ at smaller ranks and levels. Finally, we identify the number fields generated over the rationals by the entries of $S$, and by the fusion (Verlinde) eigenvalues.
\end{abstract}

\footnotetext{
* This research was supported in part by NSERC.

$\dagger$ On leave from the Physics Dept, Univ. Lethbridge, Alberta, Canada
} 


\section{Introduction}

Fix an affine non-twisted algebra $g=X_{r}^{(1)}$, and level $k$. Put $\bar{k}:=k+h^{\vee}$, where $h^{\vee}$ is the dual Coxeter number of $g$. Let $w^{0}, \ldots, w^{r}$ denote its fundamental weights, and put $\rho:=\sum_{i=0}^{r} w^{i}$. Let $P_{+}^{k}(g)$ be the set of all level $k$ integrable highest weights of $g$. For example,

$$
P_{+}^{k}\left(A_{r}^{(1)}\right)=\left\{\sum_{i=0}^{r} \lambda_{i} w^{i} \mid \lambda_{i} \in \mathbb{Z}_{\geq 0}, \sum_{i=0}^{r} \lambda_{i}=k\right\}
$$

Write $c h_{\lambda}$ for the corresponding character. Sometimes it is convenient to write $\left(\lambda_{0}, \lambda_{1}, \ldots, \lambda_{r}\right)$ for $\sum_{i} \lambda_{i} w^{i}$. When the level of a weight is known, we will often drop the $w^{0}$ component. For example, the element $k w^{0}$ of $P_{+}^{k}(g)$ will be denoted by 0 . The corresponding quantities for the underlying finite-dimensional Lie algebra $\bar{g}$ will always be denoted with a bar.

Under the familiar action of $S L_{2}(\mathbb{Z})$ on the Cartan subalgebras of $g$, we find that the span of the level $k$ characters $c h_{\lambda}$ is stable. In particular, define a matrix $S$ by:

$$
c h_{\lambda}\left(\frac{-1}{\tau}, \frac{z}{\tau}, u-\frac{(z \mid z)}{\tau}\right)=\sum_{\mu \in P_{+}^{k}(g)} S_{\lambda, \mu} c h_{\mu}(\tau, z, u) .
$$

$S$ has several interesting properties. Most importantly:

LEMma 1 (Kac-Peterson [16]): Let $\overline{c h}_{\bar{\nu}}$ denote the Weyl character of $\bar{g}$ with highest weight $\bar{\nu}$. Then for any $\lambda, \mu \in P_{+}^{k}(g)$, we have both $S_{0, \mu} \neq 0$ and

$$
\frac{S_{\lambda, \mu}}{S_{0, \mu}}=\overline{c h}_{\bar{\lambda}}\left(-2 \pi \mathrm{i} \frac{\overline{\mu+\rho}}{\bar{k}}\right)=: \chi_{\lambda}(\mu) \text {. }
$$

By Lemma 1, a useful expression for $\chi_{\lambda}(\mu)$ is

$$
\chi_{\lambda}(\mu)=\sum_{\beta \in \bar{\Omega}(\bar{\lambda})} \sum_{\gamma \in \bar{W} \beta} m_{\bar{\lambda}}(\beta) \exp \left[-2 \pi \mathrm{i} \frac{\gamma \cdot(\overline{\mu+\rho})}{\bar{k}}\right]
$$

where $\bar{W}$ is the (finite) Weyl group, where $\bar{\Omega}(\bar{\lambda})$ is the set of dominant weights of the representation of $\bar{g}$ with highest weight $\bar{\lambda}$, and where $m_{\bar{\lambda}}(\beta)$ is the weight multiplicity. 
A classical result is:

LEMma 2 (Cartan [3]): For each $\bar{\nu}$, we can write $\overline{c h}_{\bar{\nu}}=P_{\bar{\nu}}\left(\overline{c h} \overline{w^{1}}, \ldots, \overline{c h} \overline{w^{r}}\right)$ for some polynomial $P_{\bar{\nu}}\left(x_{1}, \ldots, x_{r}\right)$.

Therefore,

$$
\chi_{\lambda}(\mu)=P_{\bar{\lambda}}\left(\chi_{w^{1}}(\mu), \ldots, \chi_{w^{r}}(\mu)\right)
$$

for all $\mu \in P_{+}^{k}(g)$.

Define the fusion matrices $N_{\lambda}$ by Verlinde's formula [21]:

$$
\left(N_{\lambda}\right)_{\mu}^{\nu}:=N_{\lambda, \mu}^{\nu}=\sum_{\gamma \in P_{+}^{k}(g)} S_{\lambda, \gamma} \frac{S_{\mu, \gamma}}{S_{0, \gamma}} S_{\nu, \gamma}^{*}
$$

Equation (1.3) tells us that the $N_{\lambda}$ are simultaneously diagonalized by $S$, and have eigenvalues $\chi_{\lambda}(\mu)$. The fusion algebra (or Verlinde algebra) of $g$ at level $k$ is defined to be the $\mathbb{C}$-span of $\left\{N_{\lambda}: \lambda \in P_{+}^{k}(g)\right\}$. It is associative and commutative, with unit $N_{0}=I$ and integer structure constants $N_{\lambda, \mu}^{\nu}$ :

$$
N_{\lambda} N_{\mu}=\sum_{\nu \in P_{+}^{k}(g)} N_{\lambda, \mu}^{\nu} N_{\nu}
$$

In fact it is isomorphic as an algebra to $\mathbb{C}^{\left\|P_{+}^{k}(g)\right\|}$, defined with componentwise addition and multiplication, and so a critical ingredient here in our definition is the choice of preferred basis $\left\{N_{\lambda}: \lambda \in P_{+}^{k}(g)\right\}$. Fusion algebras (or the corresponding fusion ring) appear in many different contexts, e.g. in rational conformal field theory (RCFT) [21]. The RCFTs with fusion algebras of the type discussed here, i.e. those associated with some $g$, are known as Wess-Zumino-Witten models. Fusion algebras also appear in the study of quantum groups [19] and Hecke algebras [14] at roots of unity, Chevalley groups at nonzero characteristic [12], and quantum cohomology [22].

Call a set $\Gamma=\left\{\gamma^{1}, \ldots, \gamma^{n}\right\} \subset P_{+}^{k}(g)$ a fusion-generator if any $N_{\lambda}$ can be written as a polynomial ${ }^{1}$ in $N_{\gamma^{1}}, \ldots, N_{\gamma^{n}}$ - in other words, if for each $\lambda \in P_{+}^{k}(g)$ there is a polynomial $P_{\lambda}\left(x_{1}, \ldots, x_{n}\right)$ such that

$$
\chi_{\lambda}(\mu)=P_{\lambda}\left(\chi_{\gamma^{1}}(\mu), \ldots, \chi_{\gamma^{n}}(\mu)\right) \quad \forall \mu \in P_{+}^{k}(g) .
$$

\footnotetext{
1 By Lagrange interpolation, 'polynomial' here is equivalent to 'function'.
} 
Equivalently, $\Gamma$ is a fusion-generator ${ }^{2}$ iff for any $\lambda, \mu \in P_{+}^{k}(g)$, the only way we can have

$$
\chi_{\gamma^{\ell}}(\lambda)=\chi_{\gamma^{\ell}}(\mu) \text { for all } \ell=1, \ldots, n,
$$

is when $\lambda=\mu$.

The equivalence of the statements of (1.4a) and (1.4b) can be seen as follows. First, if (1.4b) holds, then (1.4a) implies $\chi_{\phi}(\lambda)=\chi_{\phi}(\mu)$ for all $\phi \in P_{+}^{k}(g)$. Multiplying this result by $S_{\nu, \phi}^{*}$ and summing over $\phi \in P_{+}^{k}(g)$ gives $\lambda=\mu$, by the unitarity of the matrix $S$.

In the other direction, we need to construct a polynomial $P_{\lambda}$ in $n=\|\Gamma\|$ variables, taking values $\chi_{\lambda}(\mu)$ at $m=\left\|P_{+}^{k}(g)\right\|$ distinct points. Let $\vec{x}:=\left(x_{1}, \ldots, x_{n}\right)$ denote a point in $\mathbb{C}^{n}$, and let $\vec{x}_{a}, a=1, \ldots, m$ be the points at which the required polynomial must take the values $y_{a}$. Here $x_{a, j}=\chi_{\gamma^{j}}\left(\mu^{a}\right)$ and $y_{a}=\chi_{\lambda}\left(\mu^{a}\right)$, where $a$ labels the different weights of $P_{+}^{k}(g)$. A polynomial of minimal degree satisfying the requirements can be constructed by the Lagrange interpolation formula:

$$
P(\vec{x})=\sum_{a=1}^{m} y_{a} \prod_{b=1, b \neq a}^{m} \frac{\vec{r} \cdot\left(\vec{x}-\vec{x}_{b}\right)}{\vec{r} \cdot\left(\vec{x}_{a}-\vec{x}_{b}\right)}
$$

Here $\vec{r}$ can be any (constant) vector such that $\vec{r} \cdot\left(\vec{x}_{a}-\vec{x}_{b}\right)$ vanishes iff $a=b$.

By the fusion-rank $\mathcal{R}_{k}(g)$, we mean the minimum possible cardinality $n=\|\Gamma\|$ of a fusion-generator $\Gamma$. Such a $\Gamma$ is called a fusion-basis.

Question 1: For a given $g$ and $k$, what is the fusion-rank $\mathcal{R}_{k}(g)$, and what is a fusion-basis?

This problem was studied by Di Francesco and Zuber [6]. For the applications it should suffice to get a reasonable upper bound for the fusion-rank, and to find a $\Gamma$ which realizes that bound. Incidently, it was proven in [1] that there will be a fusion potential [13] corresponding to any fusion-generator $\Gamma$.

Question 1 seems a natural one from the fusion algebra perspective, and is especially interesting considering that the fusion-rank often turns out to be surprisingly low. This analysis should have consequences for the work of Moody, Patera, Pianzola, ... on elements of finite order in a finite-dimensional Lie group (see e.g. $[18,20]$ and references therein). It has direct relevance for the classification

2 Our definition should not be confused with the 'bootstrapped' version of a fusion-generator used in $[10]$. 
of conformal field theories (more precisely, their 1-loop partition functions; see e.g. $[9,11,10])$. Our results may lead to a new presentation of the fusion algebras, along the lines of the Schubert calculus of $[13,15]$. As another example, we mention that our problem may be related to finding bases for the quantum cohomology of Grassmannians [22].

Incidentally, these fusion algebras all have a rank of one, in a sense: precisely, the Krull dimension of a fusion algebra will be one. It is not difficult to find an element $N$ of the fusion algebra in which every fusion matrix $N_{\lambda}$ will be a polynomial. These $N$ however will in general be nontrivial linear combinations of our basis vectors (1.3). For the applications we are interested in, this observation is not helpful. There is a natural basis for the fusion algebra, namely $P_{+}^{k}(g)$, and an important condition is that fusion-generators are required to be subsets of that basis.

We will address Question 1 for $g=A_{r}^{(1)}$ in Section 3. Our best lower bound for $\mathcal{R}_{r, k}:=\mathcal{R}_{k}\left(A_{r}^{(1)}\right)$ is given in Thm. 1(2); our best upper bound and smallest fusion-generator is given in Thm. 3. Cor. 1 tells us precisely when $\left\{w^{1}\right\}$ is a fusiongenerator. Cor. 2 answers Question 1 when $r$ or $k$ is small, and Conjecture 1 gives our guess for a general statement.

Another question related to this one, which we will consider in Section 4, is:

Question 2: For $g=A_{r}^{(1)}$, when is $N_{w^{1}}$ invertible?

The first fundamental weight $w^{1}$ is especially interesting, since (1.1b) and its fusion numbers $N_{w^{1}, \mu}^{\nu}$ are so simple. Incidentally, $N_{\lambda}$ is invertible iff $N_{\lambda^{\sigma}}$ is, for any Galois element $\sigma$ (see (2.6) below) - this holds in fact for any RCFT [5]. However, the inverse of a fusion matrix will only itself be a fusion matrix in the trivial cases: $\left(N_{\lambda}\right)^{-1}=N_{\mu}$ iff both $\lambda=J^{a} 0$ and $\mu=J^{-a} 0$ for some $a \in \mathbb{Z}$, where $J$ is given in (2.1b) - again the analogue holds for any RCFT. (The proof of this uses the fact that the inverse of a non-negative integer matrix can itself be integral and non-negative, only if it is a permutation matrix.)

Our best condition for $N_{w^{1}}$ being invertible is given in Thm. 6(3), while our best conditions for noninvertibility are Thms. 6(4),(5). Together, these answer Question 2 for most $r, k$. Conjecture 2 gives our guess for the general answer.

A final question, which we solve in Section 6, was asked in [4]. It is interesting because of the Galois action (2.6) on the matrix $S$ and on the fusion coefficients.

Question 3: For $A_{r}^{(1)}$, what are the number fields $K_{r, k}$ and $L_{r, k}$ generated over 
the rationals by the entries $S_{\lambda, \mu}$, and by the fusion (Verlinde) eigenvalues $\chi_{\lambda}(\mu)$, respectively?

\section{The $A_{r, k}$ Modular Matrix $S$}

For now, let us restrict attention to $A_{r, k}$ (i.e. $A_{r}^{(1)}$ at level $k$ ). Write $\bar{r}:=r+1$, $P_{+}^{r, k}:=P_{+}^{k}\left(A_{r}^{(1)}\right)$ and $\mathcal{R}_{r, k}:=\mathcal{R}_{k}\left(A_{r}^{(1)}\right)$. The symmetry group of its CoxeterDynkin diagram is the dihedral group on $\bar{r}$ elements, generated by an order 2 conjugation $C$ and an order $\bar{r}$ simple current $J$ :

$$
\begin{aligned}
& C \lambda=\lambda_{0} w^{0}+\sum_{i=1}^{r} \lambda_{r+1-i} w^{i} \\
& J \lambda=\lambda_{r} w^{0}+\sum_{i=1}^{r} \lambda_{i-1} w^{i} .
\end{aligned}
$$

These act on the $\chi_{\lambda}(\mu)$ by

$$
\begin{aligned}
\chi_{C \lambda}(\mu) & =\chi_{\lambda}(C \mu)=\chi_{\lambda}(\mu)^{*}, \\
\chi_{J^{a} \lambda}\left(J^{b} \mu\right) & =\exp [2 \pi \mathrm{i}(b t(\lambda)+a t(\mu)+k a b) / \bar{r}] \chi_{\lambda}(\mu),
\end{aligned}
$$

where

$$
t(\lambda):=\sum_{j=1}^{r} j \lambda_{j}
$$

is called the $\bar{r}$-ality. A useful relation is

$$
t\left(J^{a} \lambda\right) \equiv a k+t(\lambda) \quad(\bmod \bar{r})
$$

Another 'symmetry' of $\chi_{\lambda}(\mu)$, when $k \neq 1$, is rank-level duality [2]:

$$
\chi_{\lambda}(\mu)=\exp [2 \pi \mathrm{i} t(\lambda) t(\mu) / \bar{r} k] \widetilde{\chi}_{\tau \lambda}(\tau \mu)^{*}
$$

where $\tau \lambda$ denotes the weight in $P_{+}^{k-1, r+1}$ corresponding to the transpose (sometimes called 'conjugate') of the Young diagram of $\lambda$, after deleting any columns of length $k$ in the transposed diagram (reminder: the $i$ th row of the Young diagram of $\lambda$ has $\sum_{j=i}^{r} \lambda_{j}$ boxes). This deletion is a consequence of (2.4f) below. We will usually denote the quantities of $A_{k-1, r+1}$ with tildes. For example, $\tau w^{\ell}=\ell \widetilde{w}^{1}$.

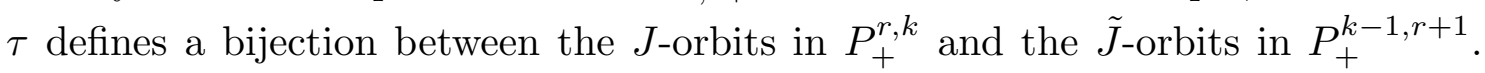
Note that

$$
\tilde{t}(\tau \lambda) \in t(\lambda)-k \mathbb{Z}_{\geq 0}
$$


since $t(\lambda)$ is the number of boxes in the Young diagram of $\lambda$.

The Weyl group of $A_{r}$ is the symmetric group $S_{\bar{r}}$. This gives us an essential property of $S$ : its relation to the symmetric polynomials. In particular, we can see from $(1.1 b)$ that

$$
\chi_{\lambda}(\mu)=\exp [2 \pi \mathrm{i} t(\lambda) t(\mu+\rho) / \bar{r} \bar{k}] S_{\lambda}\left(x_{1}, \ldots, x_{\bar{r}}\right)
$$

where $x_{\ell}:=\exp [-2 \pi \mathrm{i} \mu(\ell) / \bar{k}]$ for $\mu(\ell):=\sum_{j=\ell}^{r}\left(\mu_{j}+1\right) . S_{\lambda}$ is a polynomial over $\mathbb{Z}$ - the Schur polynomial of shape $\left(\sum_{i=1}^{r} \lambda_{i}, \sum_{i=2}^{r} \lambda_{i}, \ldots, \lambda_{r}\right)[8]-$ symmetric in the $x_{i}$, and homogeneous of degree $t(\lambda)$. It is often convenient to write $S_{\lambda}$ as a polynomial

$$
Q_{\lambda}\left(y_{1}, \ldots, y_{\bar{r} k}\right)=\sum_{m=\left(m_{1}, \ldots, m_{\bar{r} k}\right)} c_{m} \prod_{\ell} y_{\ell}^{m_{\ell}},
$$

evaluated at the 'power sums' of our $x_{i}$ :

$$
y_{\ell}=\sum_{i=1}^{\bar{r}} x_{i}^{\ell}=P_{\ell}\left(x_{1}, \ldots, x_{\bar{r}}\right) .
$$

The coefficients $c_{m}$ of $Q_{\lambda}$ can be expressed in terms of the characters of the symmetric group $S_{\bar{r}}$ (this is essentially Frobenius-Schur duality), and each nonzero $c_{m}$ will have $\sum j m_{j}=t(\lambda)[8]$. We will also write $S_{\lambda}[\mu]$ and $P_{\ell}[\mu]$, when convenient. Note that

$$
P_{\ell}\left[J^{m} \mu\right]=\exp [2 \pi \mathrm{i} \ell \mu(\bar{r}-m) / \bar{k}] P_{\ell}[\mu] .
$$

A valuable special case of $(2.4 \mathrm{a})$ is

$$
\chi_{w^{\ell}}(\mu)=\exp [2 \pi \mathrm{i} \ell t(\mu+\rho) / \bar{r} \bar{k}] \sum_{1 \leq i_{1}<\cdots<i_{\ell} \leq \bar{r}} x_{i_{1}} \cdots x_{i_{\ell}} .
$$

Symmetric polynomials have an important variable-specialisation property which permits the number of variables to be increased (with the extra variables set to 0 ), and yet all algebraic relations ${ }^{3}$ among the symmetric polynomials will be preserved. This permits us to define $\chi_{\lambda}$ when $\lambda$ has more than $\bar{r}$ components, using (2.4a) with variables $x_{1}^{\prime}=x_{1}, \ldots, x_{\bar{r}}^{\prime}=x_{\bar{r}}$, and $x_{\bar{r}+1}^{\prime}=\cdots=0$, and we find

$$
\chi_{\left(\lambda_{0}, \lambda_{1}, \ldots, \lambda_{r}, \ldots\right)}(\mu)=\left\{\begin{array}{cc}
0 & \text { if } \lambda_{\ell}>0 \text { for some } \ell>\bar{r} \\
\chi_{\left(\lambda_{0}, \lambda_{1}, \ldots, \lambda_{r}\right)}(\mu) & \text { otherwise }
\end{array}\right.
$$

3 such as (2.4), but not e.g. (2.3a), (2.6) or (2.8). More precisely, specialisation defines a homomorphism between the polynomial rings, taking Schur polynomials to Schur polynomials, power sums to power sums, etc. 
valid for any $\mu \in P_{+}^{r, k}$. This can be directly understood using for example the construction of Schur polynomials from Young Tableaux. A special case of (2.4f) is $\chi_{w^{\bar{r}}}=1$ and $\chi_{w^{\ell}}=0$ for $\ell>\bar{r}$. We will use (2.4f) in several places - see e.g. the proof of Thm. 3 .

Call $\lambda \in P_{+}^{r, k}$ a $J^{d}$-fixed point if $d$ is the smallest positive integer satisfying $J^{d} \lambda=\lambda$ - in other words if the $\lambda_{i}$ have period $d$. We will say $\lambda$ is a fixed point if it is a $J^{d}$-fixed point for some $d<\bar{r}$. Note that if $\varphi$ is a fixed point of $J^{d}$, we can speak of a 'truncated weight' $\left(\varphi_{0}, \varphi_{1}, \ldots, \varphi_{d-1}\right)=: \varphi^{\prime}$; by $(2.5 \mathrm{a})$ below it will lie in $P_{+}^{d, k d / \bar{r}}$. We have

$$
\begin{aligned}
\frac{d k}{\bar{r}} & =\sum_{i=0}^{d-1} \varphi_{i}=\sum_{i=0}^{d-1} \varphi_{i}^{\prime} \\
t(\varphi) & =\frac{\bar{r}}{d} \sum_{j=1}^{d-1} j \varphi_{j}+k \frac{\bar{r}-d}{2}=\frac{\bar{r}}{d} t^{\prime}\left(\varphi^{\prime}\right)+k \frac{\bar{r}-d}{2},
\end{aligned}
$$

where $t^{\prime}$ denotes $d$-ality. There exist $J^{d}$-fixed points in $P_{+}^{r, k}$ iff $d$ divides $\bar{r}$ and $\bar{r} / d$ divides $k$. In other words, the smallest fixed-point period is $\bar{r} / \operatorname{gcd}\{\bar{r}, k\}$, and all other possible periods are multiples of this number. Also, if $\varphi$ is a $J^{\bar{r} / d_{\text {-fixed }}}$ point, its rank-level dual $\tau \varphi$ is a $\tilde{J}^{k / d}$-fixed point.

By $(2.2 \mathrm{~b})$, if $\mu$ is a $J^{d}$-fixed point, then $\chi_{\lambda}(\mu)=0$ whenever $t(\lambda) \not \equiv 0(\bmod$ $\bar{r} / d)$. The same comment holds for $\mu$ if instead $\lambda$ is a $J^{d}$-fixed point. This is certainly not the only source of zeros in the matrix $S$ however, as we shall see, but it is an important one. In fact, there are many more zeros at fixed points than this simple $\bar{r}$-ality test suggests. For example, of all weights $\lambda$ with $t(\lambda)=$ $\bar{r} / d$, the entry $S_{\lambda, \varphi}$ will equal zero for every $J^{d}$-fixed point $\varphi$, unless $\lambda$ is a hook $\left(\frac{\bar{r}}{d}-a\right) w^{1}+w^{a}$. We will describe below the set $\mathcal{N} \mathcal{Z}(d)$ of all weights $\lambda$ which can have nonzero entries at $J^{d}$-fixed points.

Moreover, many different weights $\lambda \neq \mu$ - even in the set $\mathcal{N Z}(d)$ - will have the same value $S_{\lambda, \varphi}=S_{\mu, \varphi}$ at all $J^{d}$-fixed points $\varphi$. For example, for the hooks $\lambda$ with $t(\lambda)=\frac{\bar{r}}{d}$, we will have $\chi_{\lambda}(\varphi)= \pm \chi_{w^{\bar{r} / d}}(\varphi)$ for all $\varphi$, where the sign is independent of $\varphi$. More generally, note that the right side of (2.8c) is independent of $a^{\prime \prime}$, except for the unimportant sign.

Hence fixed point considerations are very important for both Questions 1 and 2 , and play a large role in this paper.

An unexpected symmetry of the matrix $S$ is the Galois action discussed in [5]. For any $\sigma \in \operatorname{Gal}\left(K_{r, k} / \mathbb{Q}\right)$, there exists a permutation $\mu \mapsto \sigma \mu$ of $P_{+}^{r, k}$ such 
that

$$
\begin{aligned}
\sigma S_{\lambda, \mu} & =\epsilon_{\sigma}(\mu) S_{\lambda, \sigma \mu}, \\
\sigma \chi_{\lambda}(\mu) & =\chi_{\lambda}(\sigma \mu)
\end{aligned}
$$

where $\epsilon_{\sigma}(\mu) \in\{ \pm 1\}$. Similar equations hold for any other affine algebra $g$, and more generally for any RCFT. The field $K_{r, k}$ here is generated over $\mathbb{Q}$ by all elements $S_{\lambda, \mu}$; if instead we are only interested in the permutation $\mu \mapsto \sigma \mu$, and not the 'parities' $\epsilon_{\sigma}(\mu)$, then we are more concerned with the effective Galois $\operatorname{group} \operatorname{Gal}\left(L_{r, k} / \mathbb{Q}\right)$ coming from the subfield $L_{r, k}$ generated over $\mathbb{Q}$ by the fusion eigenvalues $\chi_{\lambda}(\mu)$.

Incidentally, Galois orbits tend to be nicely behaved - see e.g. Thm. 8 below. They also have been studied in the 'elements of finite order' Lie group context see e.g. $[18,20]$.

Galois group considerations are central to many arguments in this paper, so next we will quickly review the cyclotomic Galois group. The cyclotomic field $\mathbb{Q}_{n}:=\mathbb{Q}[\exp [2 \pi \mathrm{i} / n]]$ consists of all polynomials in $\xi_{n}:=\exp [2 \pi \mathrm{i} / n]$. The Galois group $\mathcal{G}_{n}:=\operatorname{Gal}\left(\mathbb{Q}_{n} / \mathbb{Q}\right)$ is isomorphic to the multiplicative group $(\mathbb{Z} / n \mathbb{Z})^{\times}$of integers coprime to $n$, taken $\bmod n$. More precisely, any automorphism $\sigma \in \mathcal{G}_{n}$ corresponds to some integer $\ell \in(\mathbb{Z} / n \mathbb{Z})^{\times}$, in such a way that $\sigma \xi_{n}=\xi_{n}^{\ell}$. We write $\sigma_{\ell}$ for this $\sigma$. The classic example of a Galois automorphism is complex conjugation, which always corresponds to $\ell=-1$. A subfield $F$ of $\mathbb{Q}_{n}$ will have Galois $\operatorname{group} \operatorname{Gal}(F / \mathbb{Q})$ isomorphic to a factor group (equivalently here, a subgroup) of $(\mathbb{Z} / n \mathbb{Z})^{\times}$.

The previous properties of $S$ are all well known. The following one, which relates $S$ entries at fixed points to $S$ entries at both smaller rank and level, appears to be new. We will call it fixed-point factorisation.

Let $\varphi$ be a fixed point of $J^{d}$ for $A_{r, k}$. Then we will show that $\chi_{\lambda}(\varphi)=0$ unless

(*) for each $i=1, \ldots, \bar{r} / d$, there are precisely $d$ indices $\ell_{1}^{(i)}<\cdots<\ell_{d}^{(i)}$ for which $\lambda\left(\ell_{j}^{(i)}\right) \equiv-i(\bmod \bar{r} / d)$.

Assume this for now. $(*)$ implies $\frac{\bar{r}}{d}$ will divide $t(\lambda)$ - which we already know - but it is much stronger. Write $\mathcal{N} \mathcal{Z}(d)$ for the set of all weights $\lambda \in P_{+}^{r, k}$ which obey (*). We will see below that

$$
\lambda \in \mathcal{N} \mathcal{Z}(d) \quad \Longleftrightarrow \quad \chi_{\lambda}\left(\frac{k d}{\bar{r}} \sum_{i=0}^{\bar{r} / d-1} w^{d i}\right) \neq 0 .
$$


The fixed-point argument of this last equation has truncated weight $0^{\prime}$.

Consider any $\lambda \in \mathcal{N} \mathcal{Z}(d)$. Let $\pi$ be the unique permutation of $\{1, \ldots, \bar{r}\}$ defined by the following rule:

for each $1 \leq i \leq \frac{\bar{r}}{d}$ and $1 \leq j \leq d$, put $\pi\left(i+(j-1) \frac{\bar{r}}{d}\right)=\ell_{j}^{(i)}$.

$\pi$ will exist iff $(*)$ holds. For each such $i$, let $\lambda^{\prime(i)}$ denote the weight in $P_{+}^{d-1, k d / \bar{r}}$ with Dynkin labels

$$
\lambda_{j}^{\prime(i)}=\frac{\lambda\left(\ell_{j}^{(i)}\right)-\lambda\left(\ell_{j+1}^{(i)}\right)}{\bar{r} / d}-1
$$

for $j=1, \ldots, d-1$. As above, let $\varphi^{\prime} \in P_{+}^{d-1, k d / \bar{r}}$ be the truncated weight $\left(\varphi_{0}, \varphi_{1}, \ldots, \varphi_{d-1}\right)$. Then we obtain the 'factorisations'

$$
\begin{aligned}
S_{\lambda, \varphi} & =\operatorname{sgn} \pi \xi(-1)^{t(\lambda)(1-d / \bar{r})}\left(\frac{\bar{r}}{\bar{k}}\right)^{\frac{\bar{r} / d-1}{2}} S_{\lambda^{\prime}(1), \varphi^{\prime}}^{\prime} \cdots S_{\lambda^{\prime}(\bar{r} / d), \varphi^{\prime}}^{\prime} \\
\chi_{\lambda}(\varphi) & =\operatorname{sgn} \pi \xi(-1)^{t(\lambda)(1-d / \bar{r})} \chi_{\lambda^{\prime}(1)}^{\prime}\left(\varphi^{\prime}\right) \cdots \chi_{\lambda^{\prime}(\bar{r} / d)}^{\prime}\left(\varphi^{\prime}\right)
\end{aligned}
$$

where $\xi$ is the $\bar{k} d / \bar{r}$-th root of unity equal to $\exp \left[2 \pi \mathrm{i} t^{\prime}\left(\varphi^{\prime}+\rho^{\prime}\right) \sum_{i}\left(\lambda_{d}^{(i)}+i-\bar{r} / d\right)\right]$, and where primes denote quantities in $A_{d-1, k d / \bar{r}}\left(\right.$ we take $S^{\prime}=\chi^{\prime}=1$ for $d=1$ ).

Perhaps some examples at low rank and level will be helpful. For $r=3, k=4$, the only fixed points are $\left(\varphi_{0}, \varphi_{1}, \varphi_{2}, \varphi_{3}\right)=(2,0,2,0),(0,2,0,2)$, and $(1,1,1,1)$. $\mathcal{N} \mathcal{Z}(1)$ consists of the $J$-orbits of $(4,0,0,0)$ and $(2,1,0,1)$, for a total of 8 weights out of the full 35. $\mathcal{N} \mathcal{Z}(2)$ contains $\mathcal{N} \mathcal{Z}(1)$ plus the $J$-orbits of $(3,0,1,0),(2,2,0,0)$ and $(2,0,2,0)$, increasing the number of weights to 18 out of 35 . All three fixed points are in the simple-current orbits of weights of the special type indicated in (2.7a) (for $d=1$ or 2). Therefore, for these fixed points $\varphi$, we must have $S_{\varphi, \lambda} \neq 0$ for all weights $\lambda$ in the appropriate $\mathcal{N} \mathcal{Z}(d)$.

For $r=3, k=8, d=2$, however, there are fixed points such as $\varphi=(3,1,3,1)$ that are not of the type in $(2.7 \mathrm{a})$, i.e. $(4,0,4,0)$. In this case, we find that $S_{(3,1,3,1), \lambda} \neq 0$ for only 48 weights $\lambda$, while $\mathcal{N} \mathcal{Z}(2)$ has cardinality 75 (and $\left\|P_{+}^{3,8}\right\|=$ 165). The large discrepancy here between ' 48 ' and ' 75 ' is not surprising and is explained by (2.8): $\chi_{\varphi^{\prime}}^{\prime}$ will vanish at a fifth of the points of $P_{+}^{2,4}$. Incidentally, the total number of weights satisfying $t(\lambda) \equiv 0(\bmod \bar{r} / d)$ is 85 . This means there are 10 weights that satisfy the $\bar{r}$-ality test necessary for $\chi_{\lambda}(\varphi) \neq 0$, yet still have $\chi_{\lambda}(\varphi)=0$ for all $\varphi$.

Condition $(*)$ will become more severe as $r$ and $k$ increase. For example, with $r=3$ and $d=2$, the numbers of weights in $\mathcal{N} \mathcal{Z}(2)$ compared with those with even 
$\bar{r}$-ality, compared with those in $P_{+}^{3, k}$ are: 196,231 , and 455 for $k=12$; and 405 , 489, and 969 for $k=16$.

As an example of how 'factor weights' $\left\{\lambda^{\prime(i)}\right\}$ are found, consider the weight $\lambda=(0,0,1,0,0,1,1,1,0,1,1,0)$ at $r=11, k=6$. Fix $d=4$. The corresponding partition labels $\{\lambda(\ell)\}$ are $\{17,16,14,13,12,10,8,6,5,3,1,0\}$. Those congruent to $-1(\bmod \bar{r} / d=3)$ are $\{17,14,8,5\}$. From these we find $\lambda^{\prime(1)}=(1,0,1,0)$, where the zeroth Dynkin label is set so that the factor weight is at level $k d / \bar{r}=2$. We find $\lambda^{\prime(2)}=(0,0,0,2)$ and $\lambda^{\prime(3)}=(1,1,0,0)$ in similar fashion.

For a more general example, consider any hook $\lambda=a w^{1}+w^{b}$. It will lie in $\mathcal{N Z}(d)$ iff $\bar{r} / d$ divides $a+b$, in which case we find

$$
\chi_{a w^{1}+w^{b}}(\varphi)=\xi(-1)^{a+b+c+\left(a^{\prime \prime}+1\right)\left(c+a^{\prime}+1\right)} \chi_{\left(a^{\prime}-1\right) w^{\prime 1}+w^{\prime c-a^{\prime}+1}}\left(\varphi^{\prime}\right)
$$

where $c=(a+b) d / \bar{r}$ and $a=\frac{\bar{r}}{d} a^{\prime}-a^{\prime \prime}$, for $a^{\prime \prime} \in\left\{1, \ldots, \frac{\bar{r}}{d}\right\}$, and where $\xi=1$ unless $b>\bar{r}-\bar{r} / d$, in which case $\xi=\exp \left[2 \pi \mathrm{i} t^{\prime}\left(\varphi^{\prime}+\rho^{\prime}\right) \bar{r} / d \bar{k}\right]$. The permutation $\pi$ here is the product of $c-a^{\prime}+1$ disjoint $a^{\prime \prime}$-cycles. In this example, each $\lambda^{\prime(i)}=0^{\prime}$ except for $\lambda^{\left(a^{\prime \prime}\right)}=\left(a^{\prime}-1\right) w^{1}+w^{\prime c-a^{\prime}+1}$. Equation (2.8c) says that hooks in $P_{+}^{r, k}$ act like hooks in $P_{+}^{d-1, k d / \bar{r}}$, when their fusion eigenvalues are restricted to fixed points of $J^{d}$. The most interesting special case of $(2.8 \mathrm{c})$ is

$$
\chi_{w^{\ell}}(\varphi)=\left\{\begin{array}{cc}
\chi_{w^{\prime \ell d / \bar{r}}}^{\prime}\left(\varphi^{\prime}\right) & \text { if } \bar{r} / d \text { divides } \ell \\
0 & \text { otherwise }
\end{array} .\right.
$$

LEMma 3 (fixed-point factorisation): Choose any $A_{r, k}$, any divisor d of $g c d\{\bar{r}, k\}$, and any $\lambda \in P_{+}^{r, k}$. Then exactly one of the following holds:

(i) $S_{\lambda, \varphi}=\chi_{\lambda}(\varphi)=0$ for all fixed points $\varphi$ of $J^{d}$; or

(ii) $\lambda \in \mathcal{N Z}(d)$ and so $\lambda$ obeys (2.8a), (2.8b) for every fixed point $\varphi$ of $J^{d}$.

The leading signs in (2.8) are independent of $\varphi$ and so for our purposes are of no significance. The phase $\xi$ depends only on $\varphi$ and will often equal 1. Of course the right side of (2.8b) can be 'linearised' by expanding it out using fusion coefficients. Conversely, it leads to the curious observation that the fusion coefficients of $A_{r, k}$ can be seen in the fusion eigenvalues of $A_{2 r+1,2 k}$ evaluated at fixed points.

At present we do not have formulas of equal generality for the other affine algebras with simple currents. One would expect that $E_{6}^{(1)}$ would be related in this way to $G_{2}^{(1)}, E_{7}^{(1)}$ to $F_{4}^{(1)}, D_{r}^{(1)}$ for its vector simple current (i.e. the one 
interchanging $w^{0}$ and $w^{1}$, and $w^{r-1}$ and $\left.w^{r}\right)$ to $C_{r-2}^{(1)}$, etc. Perhaps an algebraic understanding of these equations can be obtained from the ideas in e.g. [7].

To prove equations (2.8), first note that

$$
P_{\ell}[\varphi]=\left\{\begin{array}{cc}
\frac{\bar{r}}{d} P_{\ell d / \bar{r}}^{\prime}\left[\varphi^{\prime}\right] & \text { if } \bar{r} / d \text { divides } \ell \\
0 & \text { otherwise }
\end{array}\right.
$$

from which we immediately obtain that $H_{\ell}[\varphi]$ equals $H_{\ell d / \bar{r}}^{\prime}\left[\varphi^{\prime}\right]$ or 0 (for $\bar{r} \mid d \ell, \bar{r} X d \ell$, respectively), for the 'complete' symmetric polynomials $H_{\ell}:=S_{\ell w^{1}}$, since (2.4b) for $\lambda=\ell w^{1}$ takes a simple form [8]. We have the determinantal formula [8]

$$
S_{\lambda}=\operatorname{det}\left(H_{\lambda(i)-\bar{r}+j}\right)_{1 \leq i, j \leq \bar{r}}=\sum_{\sigma} \operatorname{sgn} \sigma H_{\lambda(\sigma 1)-\bar{r}+1} \cdots H_{\lambda(\sigma \bar{r})-\bar{r}+\bar{r}}
$$

In this formula, $H_{0}$ identically equals 1 , and for negative $\ell, H_{\ell}$ is identically 0 . Evaluated at the fixed point $\varphi$, this will be a sparse matrix: each row will have at most $d$ nonzero elements, spaced $\bar{r} / d$ entries apart. If $S_{\lambda}[\varphi] \neq 0$, then some product $H_{\lambda(\sigma 1)-\bar{r}+1}[\varphi] \cdots H_{\lambda(\sigma \bar{r})-\bar{r}+\bar{r}}[\varphi] \neq 0$, and thus $\left\{\ell_{1}^{(i)}, \ldots, \ell_{d}^{(i)}\right\}=\{\sigma i, \sigma(i+$ $\left.\left.\frac{\bar{r}}{d}\right), \ldots, \sigma\left(i+\bar{r}-\frac{\bar{r}}{d}\right)\right\}$ for each $i$. This shows that $(*)$ is satisfied, and that the permutation $\pi$ exists. The sum in (2.9b) can be restricted to those $\sigma$ in the coset $\left(S_{d}\right)^{\times(\bar{r} / d)} \pi \subset S_{\bar{r}}$, where the $i$-th factor $S_{d}$ permutes the indices congruent to $i$ $(\bmod \bar{r} / d)$. So $(2.9 \mathrm{~b})$ can now be written as the product of determinants, the $i$-th one of which corresponds to the weight $\lambda^{\prime(i)} \in P_{+}^{d-1, k d / \bar{r}}$ (note that $(2.4 \mathrm{f}$ ) is implicit in $(2.7 \mathrm{~b})$ ), which gives us (2.8b). Equation (2.7a) follows from (2.8b) and the fact that $\left(\frac{k d}{\bar{r}} \sum_{i} w^{d i}\right)^{\prime}=0^{\prime}$. Using the product formula (= Weyl denominator formula) for $S_{0, \mu}$, we can show

$$
S_{0, \varphi}=\left(\frac{\bar{r}}{\bar{k}}\right)^{\frac{\bar{r} / d-1}{2}}\left(S_{0^{\prime}, \varphi^{\prime}}^{\prime}\right)^{\bar{r} / d}
$$

Together with (2.8b), this immediately gives us (2.8a).

\section{Fusion-rank of $A_{r, k}$}

The original polynomial realisation $[13,15]$ uses the Cartan fusion-generator $\Gamma=\left\{w^{1}, \ldots, w^{r}\right\}$, which works by Lemma 2. We can do better. From (2.2a) and Lemma 2, we see that $\mathcal{R}_{r, k} \leq \frac{\bar{r}}{2}$, with $\Gamma=\left\{w^{1}, \ldots, w^{\lfloor\bar{r} / 2\rfloor}\right\}$, where $\lfloor x\rfloor$ is the largest integer not larger than $x$. For example, the fusion-rank of $A_{1, k}$ and $A_{2, k}$ equals 1 for all $k$, with $\left\{w^{1}\right\}$ a fusion-generator. This result for $A_{2}$ was first 
obtained in [6], though by a more complicated argument. We also obtain, from Thm. 2(3) below (rank-level duality), the bound $\mathcal{R}_{r, k} \leq \frac{k}{2}+1$.

We begin by collecting a few simple consequences of the previous comments. Parts (1) and (3) of Thm. 1 are technical facts we will use repeatedly in the rest of the paper. Thm. 1(2) gives a fairly strong lower bound on $\mathcal{R}_{r, k}$. We give some consequences of Thm. 1(4) in the paragraph before Conjecture 1.

THEOREM 1 (simple-current constraints): (1) Let $\Gamma$ be a fusion-generator, and choose any $\mu \in P_{+}^{r, k}$. Let $\Gamma_{\mu}$ be the set of all $\gamma \in \Gamma$ for which $\chi_{\gamma}(\mu) \neq 0$. Let $d=\operatorname{gcd}\left\{\bar{r}, k,\left.t(\gamma)\right|_{\gamma \in \Gamma_{\mu}}\right\}$ (put $d=\bar{r}$ if $\Gamma_{\mu}=\emptyset$ ). Then $\mu$ is a $J^{\bar{r} / d}$-fixed point.

(2) (our best lower bound) Let $\Gamma$ be any fusion-generator. Write out the prime decomposition $D:=\operatorname{gcd}\{\bar{r}, k\}=\prod p_{i}^{a_{i}}$, where each prime $p_{i}$ is distinct. Then

$$
\mathcal{R}_{r, k} \geq \sum a_{i}
$$

If $D \neq \bar{r}$, we get the stronger bound

$$
\mathcal{R}_{r, k} \geq 1+\sum a_{i}
$$

More precisely, for each $p_{i}$, and each $\ell, 1 \leq \ell \leq a_{i}$, there must be some $\gamma \in \Gamma \cap \mathcal{N} \mathcal{Z}\left(\bar{r} p_{i}^{\ell} / D\right)$ (see Lemma 3) with $\operatorname{gcd}\{D, t(\gamma)\}=D / p_{i}^{\ell}$. When $D \neq \bar{r}$, there must also be some $\gamma \in \Gamma \cap \mathcal{N} \mathcal{Z}(\bar{r} / D)$ whose $\bar{r}$-ality $t(\gamma)$ is a multiple of D.

(3) Suppose $J^{\bar{r} / d} \mu=\mu$ and $J^{\bar{r} / d} \nu=\nu$ for some divisor $d$ of $\bar{r}$. Then for any weight $\lambda, \chi_{\lambda}(\mu)=\chi_{\lambda}(\nu) \neq 0$ implies $t(\lambda) t(\mu) \equiv t(\lambda) t(\nu)(\bmod d \bar{r})$.

(4) When $\bar{k}_{1}$ is some multiple of $\bar{k}_{2}$, then $\mathcal{R}_{r, k_{1}} \geq \mathcal{R}_{r, k_{2}}$.

Proof (1) Let $\mu$ be a $J^{c}$-fixed point. Then from the previous remarks, $c$ must divide $\bar{r}$, and $\bar{r} / c$ must divide both $k$ and $t(\gamma)$ for each $\gamma \in \Gamma_{\mu}$. Therefore $c$ must be a multiple of $\bar{r} / d$. Moreover $\chi_{\gamma}\left(J^{\bar{r} / d} \mu\right)=\chi_{\gamma}(\mu)$ for all $\gamma \in \Gamma_{\mu}$ (hence all $\gamma \in \Gamma$ ); since $\Gamma$ is a fusion-generator this means $J^{\bar{r} / d} \mu=\mu$, and hence $c=\bar{r} / d$.

(2) We know that for every divisor $d$ of $D$, there are $J^{\bar{r} / d}$-fixed points (more than one, unless $d=D=\bar{r}$ ). Choose such a fixed point $\varphi$, say. Let $\Gamma_{\varphi}$ be as in (1) - necessarily $\Gamma_{\varphi} \subseteq \mathcal{N} \mathcal{Z}(\bar{r} / d)$. Then, by $(1), \operatorname{gcd}\left\{\bar{r}, k,\left.t(\gamma)\right|_{\gamma \in \Gamma_{\varphi}}\right\}=d$. So we see there must be a subset $\Gamma_{d} \subseteq \Gamma$, namely $\Gamma_{d}=\Gamma_{\varphi}$, such that $\operatorname{gcd}\left\{D,\left.t(\gamma)\right|_{\gamma \in \Gamma_{d}}\right\}=d$. Note that each $\Gamma_{D / p_{i}^{\ell}}$ must contain some weight $\gamma$ with $\operatorname{gcd}\{D, t(\gamma)\}=D / p_{i}^{\ell}$

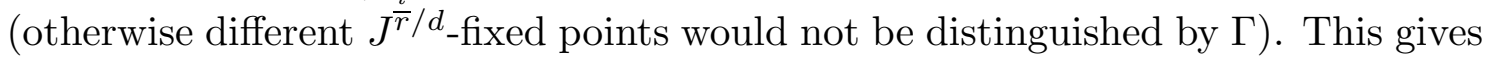


the first bound. If $\bar{r} \neq D$, then there will be several $J^{\bar{r} / D}$-fixed points, and in order for $\Gamma$ to distinguish them, $\Gamma_{D}$ must be nonempty. This gives the second bound.

(3) Let $P_{\ell}$ be the $\ell$ th power sum polynomial (2.4c). From $(2.4 \mathrm{~d})$ and $(2.5 \mathrm{a})$, $P_{\ell}[\mu] \neq 0$ requires $d$ to divide $\ell$. Consider the $m=\left(m_{1}, m_{2}, \ldots\right)$-th term in $Q_{\lambda}$ (see $(2.4 \mathrm{~b})$ ); either it will vanish at $\mu$, or $d$ will divide each $\ell$ with $m_{\ell} \neq 0$. Since $P_{\ell}[\mu]$ lies in the cyclotomic field $\mathbb{Q}[\exp [2 \pi \mathrm{i} \ell / \bar{k}]]$, we find that $S_{\lambda}[\mu]$ lies in the cyclotomic field $\mathbb{Q}[\exp [2 \pi \mathrm{i} d / \bar{k}]]$. Therefore $(2.4 \mathrm{a})$ applied to $\chi_{\lambda}(\mu)=\chi_{\lambda}(\nu) \neq 0$ gives us the desired conclusion.

(4) First note that we have the containment $\frac{\bar{k}_{1}}{\bar{k}_{2}}\left(P_{+}^{r, k_{2}}+\rho\right) \subset P_{+}^{r, k_{1}}+\rho$. Moreover, for any weight $\gamma$ we have $\chi_{\gamma}^{(1)}\left(\frac{\bar{k}_{1}}{\bar{k}_{2}}(\mu+\rho)-\rho\right)=\chi_{\gamma}^{(2)}(\mu)$ for all $\mu \in P_{+}^{r, k_{2}}$, where the superscripts indicate that $k_{1}$ or $k_{2}$ should be substituted for $k$ in $(1.1 \mathrm{~b})$. Suppose $\Gamma^{(1)}$ is a fusion-generator for $A_{r, k_{1}}$. Then for any $\mu, \nu \in P_{+}^{r, k_{2}}$, if we have $\chi_{\gamma}^{(2)}(\mu)=\chi_{\gamma}^{(2)}(\nu)$ for all $\gamma \in \Gamma^{(1)}$, then we know $\mu=\nu$. Now the $\rho$-shifted action of the affine Weyl group at level $k_{2}$ will map any weight $\gamma \in \Gamma^{(1)}$ either to some $\gamma^{\prime} \in P_{+}^{r, k_{2}}$ or onto the 'boundary' of $P_{+}^{r, k}$. In the former case we get $\chi_{\gamma}^{(2)}(\mu)= \pm \chi_{\gamma^{\prime}}^{(2)}(\mu)$, for some sign independent of $\mu$. In the latter case $\chi_{\gamma}^{(2)}(\mu)=0$ for any $\mu$, and can be ignored. Therefore, the set of weights $\gamma^{\prime}$ in $P_{+}^{r, k_{2}}$ obtained in this way from those in $\Gamma^{(1)}$ will be a fusion-generator for $A_{r, k_{2}}$.

Equation (2.3a) suggests that the fusion-generators for $A_{r, k}$ should be related to those of $A_{k-1, r+1}$. This is indeed so:

Theorem 2 (rank-level duality): (1) Suppose $\bar{r}$ does not divide $k$. Then $\mathcal{R}_{r, k} \geq$ $\mathcal{R}_{k-1, r+1}$. Moreover, if $\Gamma=\left\{\gamma^{1}, \ldots, \gamma^{n}\right\}$ is a fusion-generator for $A_{r, k}$, then $\widetilde{\Gamma}$ $=\left\{\tilde{J}^{a_{1}} \tau \gamma^{1}, \ldots, \tilde{J}^{a_{n}} \tau \gamma^{n}\right\}$ is one for $A_{k-1, r+1}$, where each $a_{i}$ is chosen so that $\operatorname{gcd}\left\{a_{i} \bar{r}+t\left(\gamma^{i}\right), k\right\}=\operatorname{gcd}\left\{t\left(\gamma^{i}\right), \bar{r}, k\right\}$ for each $i$.

(2) If $\bar{r}$ does not divide $k$, and $k$ does not divide $\bar{r}$, then $\mathcal{R}_{r, k}=\mathcal{R}_{k-1, r+1}$; in this case if $\Gamma$ is a fusion-basis for $A_{r, k}$, then $\widetilde{\Gamma}$, defined in (1), will be one for $A_{k-1, r+1}$.

(3) If $\bar{r}$ does divide $k$, then $\mathcal{R}_{r, k} \leq \mathcal{R}_{k-1, r+1} \leq \mathcal{R}_{r, k}+1$. Using the notation of (1), $\left\{\tilde{J} \tilde{0}, \tau \gamma^{1}, \ldots, \tau \gamma^{n}\right\}$ is a fusion-generator for $A_{k-1, r+1}$.

Proof (1) Any weight of $P_{+}^{k-1, r+1}$ can be expressed as $\tilde{J}^{b} \tau \mu$ for some integer $b$ and some weight $\mu \in P_{+}^{r, k}$. So, it suffices to consider any $\mu, \mu^{\prime} \in P_{+}^{r, k}$ and $b \in \mathbb{Z}$ for which

$$
\tilde{\chi}_{\tilde{J}^{a_{i}} \tau \gamma^{i}}(\tau \mu)=\tilde{\chi}_{\tilde{J}^{a_{i}} \tau \gamma^{i}}\left(\tilde{J}^{b} \tau \mu^{\prime}\right) \quad \forall i
$$


and show that this implies $\tau \mu=\tilde{J}^{b} \tau \mu^{\prime}$. (3.1a) becomes

$$
\chi_{\gamma^{i}}(\mu)=\exp \left[2 \pi \mathrm{i}\left\{\bar{r} a_{i}+t\left(\gamma^{i}\right)\right\}\left\{t(\mu)-t\left(\mu^{\prime}\right)-\bar{r} b\right\} / \bar{r} k\right] \chi_{\gamma^{i}}\left(\mu^{\prime}\right) .
$$

Define $\Gamma_{\mu}$ as in Thm. 1(1). Because $\bar{r}$ does not divide $k$, we know $\Gamma_{\mu} \neq \emptyset$. Equation (3.1b) and Thm. 1(1) imply that $\mu$ and $\mu^{\prime}$ will both be $J^{\bar{r} / d}$-fixed points, where $d=\operatorname{gcd}_{\gamma^{i} \in \Gamma_{\mu}}\left\{a_{i} \bar{r}+t\left(\gamma^{i}\right), k\right\}$. Then $\tau \mu$ and $\tilde{J}^{b} \tau \mu^{\prime}$ will both be $\tilde{J}^{k / d}$-fixed points. For each $\gamma^{i} \in \Gamma_{\mu}$, Thm. 1(3) and (3.1a) imply

$$
\left\{\bar{r} a_{i}+t\left(\gamma^{i}\right)\right\}\left\{t(\mu)-t\left(\mu^{\prime}\right)-\bar{r} b\right\} \equiv 0 \quad(\bmod d k) .
$$

For each prime $p \mid k$, write $p^{a}$ and $p^{a^{\prime}}$ for the exact powers dividing $k$ and $d$, respectively: i.e. $p^{a} \| k$ and $p^{a^{\prime}} \| d$. So $a \geq a^{\prime}$. If $a=a^{\prime}$, then $p^{a}$ must divide both $\bar{r}$ and $t(\mu)-t\left(\mu^{\prime}\right)$, by (2.5b). If $a>a^{\prime}$, then $p^{a^{\prime}} \|\left(\bar{r} a_{i}+t\left(\gamma^{i}\right)\right)$, for some $\gamma^{i} \in \Gamma_{\mu}$. Therefore (3.1c) tells us that $L:=\left\{t(\mu)-t\left(\mu^{\prime}\right)-\bar{r} b\right\} / k$ is an integer. Equation (3.1b) then implies $\chi_{\gamma^{i}}(\mu)=\chi_{\gamma^{i}}\left(J^{L} \mu^{\prime}\right)$ for all $i$. Therefore $\mu=J^{L} \mu^{\prime}$, so we may take $\mu=\mu^{\prime}$ in (3.1a), and absorb the $L$ into $b$. Then $\bar{r} / d$ must divide $L$, i.e. $k / d$ must divide $b$, i.e. $\tilde{J}^{b} \tau \mu=\tau \mu$, and we see that (3.1a) can only be trivially satisfied. Hence $\mathcal{R}_{k-1, r+1} \leq \mathcal{R}_{r, k}$.

(2) is immediate from part (1).

(3) The first inequality comes from (1). That the given set is a fusiongenerator follows by the proof of (1). More precisely, by replacing $\tilde{J}^{a_{i}} \tau \gamma^{i}$ with $\tilde{J} \tilde{0}$ in (3.1a) implies $L \in \mathbb{Z}$. The rest of the argument is as before.

The Chinese Remainder Theorem tells us that it is always possible to choose the $a_{i}$ 's in Thm. 2(1). Incidentally, in all cases of which we know, $\mathcal{R}_{k-1, r+1}=$ $1+\mathcal{R}_{r, k}$ when $\bar{r}<k$ divides $k$.

Earlier we suggested the upper bound $\mathcal{R}_{r, k} \leq \bar{r} / 2$, and now we also know $\mathcal{R}_{r, k} \leq \frac{k}{2}+1$ (or $k / 2$ if $k$ fails to divide $\bar{r}$ ). In fact we can do much better than this for most pairs $(r, k)$. The argument relies on the cyclotomic Galois group $\mathcal{G}_{n}$ described briefly in the previous section.

Theorem 3 (Galois considerations): $\quad \Gamma_{\div}:=\left\{w^{d}: 2 d \leq \bar{r}\right.$ and $d$ divides $\left.\bar{k}\right\}$ is a fusion-generator for $A_{r, k}$, called the divisor generator. A related fusion-generator is $\Gamma_{\leftarrow}^{\tau}$, defined by

$$
\Gamma_{\div}^{\tau}:=\left\{\begin{array}{cc}
\left\{w^{d}: 2 d \leq k \text { and } d \text { divides } \bar{k}\right\} & \text { when } k \text { does not divide } \bar{r} \\
\left\{w^{k}\right\} \cup\left\{w^{d}: 2 d \leq k \text { and } d \text { divides } \bar{k}\right\} & \text { when } k \text { divides } \bar{r}
\end{array}\right.
$$


Moreover, each $w^{d}$ in $\Gamma_{\div}$and $\Gamma_{\div}^{\tau}$ can be replaced with any hook $\ell w^{1}+w^{d-\ell}$ for $1 \leq \ell \leq d$

Proof The key observation here is that, because each $x_{j}$ is a $\bar{k}$-th root of unity, for any $\ell$ there will exist a Galois automorphism $\sigma \in \mathcal{G}_{\bar{k}}$ for which

$$
\sigma P_{d}\left(x_{1}, \ldots, x_{\bar{r}}\right)=P_{\ell}\left(x_{1}, \ldots, x_{\bar{r}}\right)
$$

where $d=\operatorname{gcd}\{\ell, \bar{k}\}$.

Suppose, for all $d \leq \bar{r} / 2$ dividing $\bar{k}$, that

$$
\chi_{w^{d}}(\mu)=\chi_{w^{d}}\left(\mu^{\prime}\right)
$$

We will show this implies $\mu=\mu^{\prime}$. (3.3a) and (2.4a) give

$$
S_{w^{d}}[\mu]=\xi^{d} S_{w^{d}}\left[\mu^{\prime}\right]
$$

for all $d \leq \bar{r} / 2$ dividing $\bar{k}$, where $\xi=\exp \left[-2 \pi \mathrm{i}\left(t(\mu)-t\left(\mu^{\prime}\right)\right) / \bar{r} \bar{k}\right]$.

Equation (2.4b) reads

$$
S_{w^{\ell}}[\mu]=\frac{(-1)^{\ell+1}}{\ell} P_{\ell}[\mu]+\dot{Q}_{\ell}\left(P_{1}[\mu], \ldots, P_{\ell-1}[\mu]\right)
$$

for some polynomial $\dot{Q}_{\ell}$ homogeneous in the same sense as $Q_{\lambda}$ (and so has no constant term). Let $d$ be the smallest $\ell$ with $P_{\ell}[\mu] \neq 0$. Then (3.4a) implies $S_{w^{\ell}}[\mu]=0$ for all $\ell<d$ and $S_{w^{d}}[\mu]= \pm \frac{1}{d} P_{d}[\mu] \neq 0$, so either $d=\bar{r}$ (in which case $\left.\mu=\left(\frac{k}{\bar{r}}, \frac{k}{\bar{r}}, \ldots, \frac{k}{\bar{r}}\right)=\mu^{\prime}\right)$, or $d \leq \bar{r} / 2$ by $(2.2 \mathrm{a})$. But (3.2) requires $d$ to divide $\bar{k}$, if it is to be minimal. Thus (3.3b) holds. However both $S_{w^{d}}[\mu]$ and $S_{w^{d}}\left[\mu^{\prime}\right]$ lie in $\mathbb{Q}_{\bar{k} / d}$, so $\xi$ must be a $\bar{k}$-th root of unity.

We next want to show, by induction on $\ell$, that

$$
P_{\ell}[\mu]=\xi^{\ell} P_{\ell}\left[\mu^{\prime}\right]
$$

for all $\ell \leq \bar{r} / 2$. If we could show this, we would be done, because by (3.4a) it would force $\chi_{w^{\ell}}(\mu)=\chi_{w^{\ell}}\left(\mu^{\prime}\right)$ for all $\ell \leq \bar{r} / 2$, i.e. $\mu=\mu^{\prime}$. (3.4b) is clearly true for $P_{1}=S_{1}$, using (3.3b) with $d=1$. By (3.2), it is then true for all $\ell$ with $\operatorname{gcd}\{\ell, \bar{k}\}=1$. Take any divisor $d \leq \bar{r} / 2$ of $\bar{k}$, and suppose (3.4b) is true for all $\ell<d$. Using (3.3b), equation (3.4a) means that (3.4b) is true for $\ell=d$, and 
hence all $\ell$ with $\operatorname{gcd}\{\ell, \bar{k}\}=d$. Therefore (3.4b) is indeed true for all $\ell \leq \bar{r} / 2$, and $\mu=\mu^{\prime}$.

The above remarks continue to hold if we replace each $w^{d}$ with any hook $\ell w^{1}+w^{d-\ell}$ (of all the weights $\lambda$ with $t(\lambda)=d$, only the hooks have the variable $y_{d}$ appearing nontrivially in the corresponding polynomial $Q_{\lambda}\left(y_{i}\right)$ - see e.g. p.51 of [8]). Thm. 2 applied to $\Gamma_{\div}$gives us the fusion-generator $\Gamma_{\div}^{\tau}$ (the hooks $d w^{1}$ and $J 0=k w^{1}$ can be replaced here with $w^{d}$ and $w^{k}$, respectively).

In many special cases, most notably Cor. 1 and Cor. 2 below, we can prove that the divisor generator $\Gamma \div$ is actually a fusion-basis. Another example: suppose $\operatorname{gcd}\{\bar{r}, k\}=p^{\ell}$ for some prime $p$, so $\bar{k}$ will equal $p^{m} q$ for some $m \geq \ell$ and some number $q$ coprime to $p$. If all prime divisors of $q$ are larger than $\bar{r} / 2$, then $\Gamma \div$ will be a fusion-basis, and $\mathcal{R}_{r, k}=\ell+1$ (if $\bar{r} \neq p^{\ell}$ ) or $\mathcal{R}_{r, k}=\ell$ (if $\bar{r}=p^{\ell}$ ). The reason is that here the lower bound for $\mathcal{R}_{r, k}$ from Thm. 1(2) agrees with the upper bound from Thm. 3. A special case of this occurs when both $\bar{r}$ and $\bar{k}$ are powers of $p$.

In fact we know of only a few examples (for $\bar{r} \leq k$ ) where the divisor generator is not a fusion-basis. For $r=4$, for example, we find by computer that the fusionrank is one for $k=5,9,17$ and 21. On the other hand, the computer program tells us that the fusion-rank is 2 for $r=4$ and $k=7,11,13$ and 15 . This implies, by Thm. 1(4), that whenever $\bar{k}$ is a multiple of $12,16,18$ or $20, \mathcal{R}_{4, k}=2$ and $\Gamma \div$ will be a fusion-basis.

Conjecture 1: At fixed rank $r$, the divisor generator $\Gamma \div$ is a fusion-basis for all sufficiently high levels $k$.

For reasons of simplicity, the case of greatest interest is when $\Gamma=\left\{w^{1}\right\}$ is a fusion-generator. The complete solution to this is a consequence of this theorem:

TheOREM 4: $\quad \Gamma=\left\{w^{1}, w^{2}, \ldots, w^{m}\right\}$ is a fusion-generator of $A_{r, k}$ iff $\Gamma \div \subseteq$ or $\Gamma_{\div}^{\tau} \subseteq \Gamma$.

Proof ' $\Leftarrow$ ' is immediate from Thm. 3 .

$' \Rightarrow \quad$ Suppose we could find a polynomial

$$
p(x)=x^{m_{1}}+\cdots+x^{m_{\ell}}-x^{n_{1}}-\cdots-x^{n_{\ell}},
$$

not identically 0 , such that:

(a) $\ell<\bar{r}$, 
(b) $1 \leq m_{1}<\cdots<m_{\ell}<\bar{k}$ and $1 \leq n_{1}<\cdots<n_{\ell}<\bar{k}$,

(c) $x=\exp [2 \pi \mathrm{i} a / \bar{k}]$ is a root of $p(x)$, for each $a=1,2, \ldots, m$, and

(d) $\sum_{i=1}^{\ell} m_{i}=\sum_{i=1}^{\ell} n_{i}$.

Then there would exist weights $\lambda \neq \mu$ in $P_{+}^{r, k}$ obeying $\chi_{w^{a}}(\lambda)=\chi_{w^{a}}(\mu)$ for each $a=1, \ldots, m$ - in other words, $\Gamma$ could not in this case be a fusion-generator. To see this, choose any $\bar{r}-\ell$ distinct integers $h_{i}$ such that $h_{1}=0$, the remaining $h_{i}$ obey $1 \leq h_{i}<\bar{k}$, and $\left\{h_{i}\right\} \cap\left\{m_{i}\right\}=\left\{h_{i}\right\} \cap\left\{n_{i}\right\}=\emptyset$. The $h_{i}$ and $m_{j}$ together equal the $\bar{r}$ values of $\lambda(i)$, and the $h_{i}$ and $n_{j}$ together equal the $\bar{r}$ values of $\mu(i)$. Since $p(x) \not \equiv 0$, we know $\mu \neq \lambda$. Condition (c) says that $P_{a}[\lambda]=P_{a}[\mu]$ for all $a \leq m$, and $(\mathrm{d})$ is just the statement that $t(\lambda+\rho)=t(\mu+\rho)$. Hence $(\mathrm{c})$ together with induction on (3.4a) is equivalent to saying $\chi_{w^{a}}(\lambda)=\chi_{w^{a}}(\mu)$ for those $a$, and we are done.

It is easy to find this polynomial in many cases. In particular, let $d$ be the largest divisor of $\bar{k}$ with $2 d \leq \min \{\bar{r}, k\}$, and assume $d>m$. Take $p(x)$ to be $\left(x^{4}-x^{3}-x^{2}+x\right)\left(x^{\bar{k}-n}+x^{\bar{k}-2 n}+\cdots+x^{n}+1\right)$ where $n=\bar{k} / d$. Then (c) and (d) are automatically satisfied. $\ell=2 d$ here, so (a) will be satisfied unless $d=\bar{r} / 2$. Also, (b) will be satisfied unless $n \leq 4$, which can only happen if $d=\bar{r} / 2=k / 2$.

This argument breaks down only when $d=\bar{r} / 2$. However, when $\bar{r} / 2$ divides $\bar{k}$, there will be $J^{2}$-fixed points, and by Thm. 1(2) we would require some $\gamma \in \Gamma$ with $t(\gamma)$ a multiple of $\bar{r} / 2$ if $\Gamma$ is to be a fusion-generator.

The ony remaining way $\Gamma$ could fail to contain $\Gamma_{\div} \cap \Gamma_{\div}^{\tau}$ is if simultaneously $k \mid \bar{r}, \bar{r} \neq k$, and $m<k$. But then Thm. 1(2) applies, and $\Gamma$ would not be able to distinguish the $J^{\bar{r} / k}$-fixed points.

COROLlary 1 (the first-fundamental generator): $\quad \Gamma=\left\{w^{1}\right\}$ is a fusion-generator iff both:

(i) each prime divisor $p$ of $\bar{k}$ satisfies $2 p>\min \{\bar{r}, k\}$, and

(ii) either $\bar{r}$ divides $k$, or $\operatorname{gcd}\{\bar{r}, k\}=1$.

Incidently, the proof of Thm. 4 also implies that at least one weight $\gamma$ in any fusion-generator must have $t(\gamma) \geq d$, where $d$ is the largest divisor of $\bar{k}$ with $d \leq \bar{r} / 2$ and $d \leq k / 2$. If this $\gamma$ is not a hook, then in fact $t(\gamma)$ would have to be strictly larger than $d$.

Corollary 2: Some fusion-bases for $A_{r, k}$ are:

- $\Gamma \div=\left\{w^{1}\right\}$ for $r=1$ and $2, \forall k \geq 1$; 
- $\Gamma_{\div}=\left\{w^{1}\right\}$ for $r=3$ when $k$ is odd; $\Gamma \div=\left\{w^{1}, w^{2}\right\}$ for $r=3$ when $k$ is even;

- $\Gamma_{\div}^{\tau}=\left\{w^{1}\right\}$ for $k=1, \forall r \geq 1$;

- $\Gamma_{\leftarrow}^{\tau}=\left\{w^{1}\right\}$ for $k=2$ and any even $r$; both $\Gamma=\left\{J 0, w^{1}\right\}$ and $\Gamma_{\div}^{\tau}=\left\{w^{1}, w^{2}\right\}$ for $k=2$ and any odd $r>1$;

- $\Gamma_{\div}^{\tau}=\left\{w^{1}\right\}$ for $k=3$ and any $\bar{r}$ coprime to 3 ; both $\Gamma=\left\{J 0, w^{1}\right\}$ and $\Gamma_{\leftarrow}^{\tau}=\left\{w^{1}, w^{3}\right\}$ for $k=3$ and any multiple $\bar{r}>3$ of 3 ;

- $\Gamma_{\leftarrow}^{\tau}=\left\{w^{1}\right\}$ for $k=4$ when $r$ is even; $\Gamma_{\doteqdot}=\left\{w^{1}, w^{2}\right\}$ for $k=4$ when $r \equiv 1$ $(\bmod 4), r>4$; and both $\Gamma=\left\{J 0, w^{1}, w^{2}\right\}$ and $\Gamma_{\leftarrow}^{\tau}=\left\{w^{1}, w^{2}, w^{4}\right\}$ for $k=4$ when $r \equiv 3(\bmod 4), r>4$.

Cor. 2 follows immediately from Thm. 1(2) and Thm. 3. Some of these fusionbases are collected in the Table. Cor. 2 tells us the fusion-rank when either $r \leq 3$ or $k \leq 4$.

In addition, other fusion-bases are $\Gamma_{\div}=\left\{w^{1}\right\}$ for $r=4$ when $k$ is even, for $r=5$ when $k$ is coprime to 6 , and $\Gamma_{\div}^{\tau}=\left\{w^{1}\right\}$ for $k=6$ when $\bar{r}$ is coprime to 6 ; $\Gamma_{\div}=\left\{w^{1}, w^{2}\right\}$ for $r=5$ when $k \equiv 2,4(\bmod 6)$, and $\Gamma_{\div}^{\tau}=\left\{w^{1}, w^{2}\right\}$ for $k=6$ when $r \equiv 1,3(\bmod 6) ;$ and $\Gamma_{\div}=\left\{w^{1}, w^{3}\right\}$ for $r=5$ when $k \equiv 3(\bmod 6)$, and $\Gamma_{\div}^{\tau}=\left\{w^{1}, w^{3}\right\}$ for $k=6$ when $r \equiv 2(\bmod 6)$. The simplest cases we do not yet know the answer for are: $r=4$ when $k$ is odd $(\mathcal{R} \leq 2) ; r=5$ when 6 divides $k$ $(\mathcal{R}=2$ or 3$) ; k=5$ when $r$ is even $(\mathcal{R} \leq 3)$; and $k=6$ when 6 divides $\bar{r}(\mathcal{R}=3$ or 4$)$.

Obviously to go further we need a better lower bound. Thm. 1(2) is the best we have, but it only exploits the presence of fixed points.

\section{The fusion matrix of $w^{1}$}

There are many times when it is useful to know whether particular $S$ matrix elements are nonzero. This is the case for example in almost every modular invariant partition function classification attempt - e.g. see the underlying assumption in [17]. It is especially useful to answer this for the first fundamental weight $w^{1}-$ in Thm. 5 below we give some consequences.

For later convenience, define the sets

$$
\begin{aligned}
\mathcal{P}_{r, k} & :=\{p \text { prime }: p \leq \min \{\bar{r}, k\} \text { and } p \text { divides } \bar{k}\} \\
\mathbb{Z}_{\geq} X & :=\left\{\sum_{x \in X} a_{x} x: a_{x} \in \mathbb{Z}_{\geq 0}\right\}
\end{aligned}
$$




\begin{tabular}{|c|c|c|c|c|c|}
\hline$r \backslash k$ & 1 & 2 & 3 & 4 & 5 \\
\hline 1 & $\overline{\left|\left\{w^{1}\right\}\right|} \uparrow$ & $\left\{w^{1}\right\}$ & $\left\{w^{1}\right\} \uparrow$ & $\left\{w^{1}\right\}$ & $\left\{w^{1}\right\} \uparrow$ \\
\hline 2 & $\left|\left\{w^{1}\right\}\right| \uparrow$ & $\left\{w^{1}\right\} \uparrow$ & $\left\{w^{1}\right\}$ & $\left\{w^{1}\right\} \uparrow$ & $\left\{w^{1}\right\} \uparrow$ \\
\hline 3 & $\mid\left\{w^{1}\right\} \uparrow$ & $\overline{\left\{w^{1}, w^{2}\right\}} \mid$ & $\left\{w^{1}\right\} \uparrow$ & $\left\{w^{1}, w^{2}\right\}$ & $\left\{w^{1}\right\} \uparrow$ \\
\hline 4 & $\left\{w^{1}\right\} \uparrow$ & $\left\{w^{1}\right\} \uparrow$ & $\left\{w^{1}\right\} \uparrow$ & $\left\{w^{1}\right\} \uparrow$ & $\left\{w^{2}\right\}$ \\
\hline 5 & $\left\{w^{1}\right\} \uparrow$ & $\left\{w^{1}, w^{2}\right\}$ & $\overline{\left\{w^{1}, w^{3}\right\}}$ & $\overline{\left\{w^{1}, w^{2}\right\}}$ & $\left\{w^{1}\right\} \uparrow$ \\
\hline 6 & $\left\{w^{1}\right\} \uparrow$ & $\left\{w^{1}\right\} \uparrow$ & $\left\{w^{1}\right\} \uparrow$ & $\left\{w^{1}\right\} \uparrow$ & $\left\{w^{1}, w^{2}\right\}$ \\
\hline 7 & $\left\{w^{1}\right\} \uparrow$ & $\left\{w^{1}, w^{2}\right\}$ & $\left\{w^{1}\right\} \uparrow$ & $\left\{w^{1}, w^{2}, w^{4}\right\}$ & $\left\{w^{1}\right\} \uparrow$ \\
\hline 8 & $\left\{w^{1}\right\} \uparrow$ & $\left\{w^{1}\right\} \uparrow$ & $\left\{w^{1}, w^{3}\right\}$ & $\left\{w^{1}\right\} \uparrow$ & $\left\{2 w^{2}+w^{5}\right\} \uparrow$ \\
\hline
\end{tabular}

Table. Listed are $A_{r, k}$ fusion-bases for low ranks and/or levels. The symbols | in rows of the Table delimit sequences of fusion-bases that repeat indefinitely as the level $k$ increases. For increasing ranks $r$, overlines and underlines work similarly in the columns. ' $\uparrow$ ' signifies that $N_{w^{1}}$ is invertible (see Section 4).

where $X$ in (4.1b) is any set of natural numbers. $\mathbb{Z}_{\geq} X$ is the set of all possible sums (repetitions allowed) of elements of $X$. For example, $\mathbb{Z}_{\geq}\{n\}=\{0, n, 2 n, \ldots\}$ is the set of all nonnegative multiples of $n$.

Theorem 5: (1) Suppose $S_{w^{1}, \mu}=0$. Then $S_{\lambda, \mu}=0$ unless $t(\lambda) \in \mathbb{Z}_{\geq} \mathcal{P}_{r, k}$. Both $k$ and $\bar{r}$ must lie in $\mathbb{Z}_{\geq} \mathcal{P}_{r, k}$.

(2) Suppose there is only one prime divisor $p$ of $\bar{k}$ not larger than $\min \{\bar{r}, k\}$. Then $S_{w^{1}, \mu}=0$ iff $\mu$ is a fixed point.

Proof When $k \geq \bar{r}$, part (1) follows by considering the polynomial expression (2.4b) and using the Galois argument of (3.2): $P_{\ell}[\mu] \neq 0$ requires $\ell \in \mathbb{Z}_{\geq} \mathcal{P}_{r, k}$. Taking $\lambda=J 0$ gives us $k \in \mathbb{Z}_{\geq} \mathcal{P}_{r, k}$, and $\lambda=w^{\bar{r}}$ (see (2.4f)) gives us $\bar{r} \in \mathbb{Z}_{\geq} \mathcal{P}_{r, k}$. When $k<\bar{r}$, to show that we can restrict to primes $p \leq k$, we use rank-level duality (2.3a) to get that $\tilde{t}(\tau \lambda) \in \mathbb{Z}_{\geq} \mathcal{P}_{r, k}$ and then $t(\lambda) \in \mathbb{Z}_{\geq} \mathcal{P}_{r, k}$ follows from (2.3b) and the fact that $k \in \mathbb{Z}_{\geq} \mathcal{P}_{r, k}$. For part (2), use part (1) and Thm. 1(1) to get that $\mu$ must be fixed by $J^{\bar{r} / p}$. 
Note that the hypothesis of (2) holds whenever $\bar{k}$ is a power of a prime. This special case follows directly from (4.2) below, by using Gauss' Lemma on factorising integral polynomials, and evaluating certain factored polynomials at 1 . Thm. 5(2) however is much more general.

$N_{w^{1}}$ is invertible iff $S_{w^{1}, \mu} \neq 0$ for all $\mu \in P_{+}^{r, k}$. Equivalently, $N_{w^{1}}$ is invertible iff

$$
\sum_{j=1}^{\bar{r}} \exp [2 \pi \mathrm{i} \mu(j) / \bar{k}] \neq 0 \quad \forall \mu \in P_{+}^{r, k} .
$$

It is not hard to show that for $k \leq 4$ or $\bar{r} \leq 4, N_{w^{1}}$ is invertible iff $\operatorname{gcd}\{\bar{r}, k\}=1$; in fact, for those $r, k, \chi_{w^{1}}(\mu)=0$ only for fixed points $\mu$. The identical conclusion holds for many other $r$ and $k$, as we saw in Thm. 5(2). But Thms. 6(4),(5) below say that these cases are uncharacteristically well-behaved. For example, when $\bar{r}=5$, if 6 divides $\bar{k} \geq 12$, then $N_{w^{1}}$ will not be invertible, even though there are no fixed points.

THEOREM 6 (invertibility): (1) $N_{w^{1}}$ is invertible iff $\tilde{N}_{\tilde{w}^{1}}$ is, where the latter is the fusion matrix for $A_{k-1, r+1}$.

(2) If $\operatorname{gcd}\{\bar{r}, k\} \neq 1$, then $N_{w^{1}}$ cannot be invertible.

(3) $N_{w^{1}}$ is invertible if either $\bar{r} \notin \mathbb{Z}_{\geq} \mathcal{P}_{r, k}$ or $k \notin \mathbb{Z}_{\geq} \mathcal{P}_{r, k}$.

(4) Suppose $p q$ divides $\bar{k}$, where $p$ and $q$ are distinct primes for which $\bar{r} \in \mathbb{Z}_{\geq}\{p, q\}$ - i.e. there exist nonnegative integers $a, b$ such that ap $+b q=\bar{r}$. If $\bar{k} \geq$ $p q\left(\left\lceil\frac{a}{q}\right\rceil+\left\lceil\frac{b}{p}\right\rceil\right)$, then $N_{w^{1}}$ will not be invertible $(\lceil x\rceil$ here denotes the smallest integer not smaller than $x-$ e.g. $\lceil 2\rceil=2,\lceil 3.1\rceil=4)$.

(5) Suppose $p_{1}, p_{2}, \ldots, p_{n}$ are primes dividing $\bar{k}$ for which $\bar{r} \in \mathbb{Z}_{\geq}\left\{p_{1}, \ldots, p_{n}\right\}$ - i.e. there exist nonnegative integers $a_{i}$ such that $\sum a_{i} p_{i}=\bar{r}$. If $\bar{k} \geq$ $p_{i} p_{j} \sum_{h=i}^{j} a_{h}$ for any $i<j$, then $N_{w^{1}}$ will not be invertible.

Proof (1) follows directly from (2.3a). (2) exploits the fact (see (2.2b)) that $\chi_{w^{1}}(\varphi)=0$ for any fixed point $\varphi$. (3) is a corollary of Thm. 5(1).

(4) We want to construct a particular $\mu \in P_{+}^{r, k}$ such that $\chi_{w^{1}}(\mu)=0$. To do this we find an arithmetic sequence $\frac{\bar{k}}{p} \mathbb{Z}+c_{i}$ for each $i=1, \ldots, a$, and an arithmetic sequence $\frac{\bar{k}}{q} \mathbb{Z}+c_{j}^{\prime}$ for each $j=1, \ldots, b$, such that none of these $a+b$ sequences intersect. This is easy to do, provided $\bar{k}$ is big enough. Choose as the $c_{i}$ 's $0, \frac{\bar{k}}{q}, \ldots, \frac{\bar{k}}{q}(q-1), 1,1+\frac{\bar{k}}{q}, \ldots, 1+\frac{\bar{k}}{q}(q-1)$, etc, until we have chosen $a$ of them (the last one will be $\left\lceil\frac{a}{q}\right\rceil-1$ plus some multiple of $\frac{\bar{k}}{q}$ ). Next choose as the $c_{j}^{\prime}$ 's 
$\left\lceil\frac{a}{q}\right\rceil,\left\lceil\frac{a}{q}\right\rceil+\frac{\bar{k}}{p}, \ldots$, until we have chosen $b$ of them (the last one will be $\left\lceil\frac{a}{q}\right\rceil+\left\lceil\frac{b}{p}\right\rceil-1$ plus some multiple of $\frac{\bar{k}}{p}$ ). Our $a+b$ sequences will be disjoint, provided the bound on $\bar{k}$ is satisfied, and will intersect the interval $0 \leq x<\bar{k}$ in precisely $a p+b q=\bar{r}$ points. Let $\mu$ be the unique weight in $P_{+}^{r, k}$ whose $\mu(\ell)$ equal those $\bar{r}$ points. Then $\chi_{w^{1}}(\mu)=0$, because the sum in (4.2) along each of the $a+b$ sequences is 0 .

(5) follows immediately from similar considerations: we are looking for $a_{i}$ series $\frac{\bar{k}}{p_{i}} \mathbb{Z}+c_{i j}$, where $c_{i j} \not \equiv c_{i \ell}\left(\bmod \frac{\bar{k}}{p_{i}}\right)$ for $j \neq \ell$, and $c_{i j} \not \equiv c_{h \ell}\left(\bmod \frac{\bar{k}}{p_{i} p_{h}}\right)$ for $i \neq h$. The choice $c_{i j}=j-1+\sum_{\ell=1}^{i-1} a_{\ell}$ works.

The proofs of Thms. 6(4),(5) are constructive: their zeros arise when (4.2) finds itself a sum of terms such as $\sum_{a=1}^{p} \xi^{a}$ for $\xi$ a primitive $p$-th root of unity. A simple example of Thm. $6(4)$ is at $\bar{r}=11, \bar{k}=30$. With $p=3, q=5$, and $a=2, b=1$, the bound is saturated. One finds $c_{1}=0, c_{2}=6$ and $c_{1}^{\prime}=1$. These yield $0,10,20 ; 6,16,26$; and $1,7,13,19,25$; respectively. So, there is a zero for the weight given by $\{\mu(1), \ldots, \mu(\bar{r})\}=\{26,25,20,19,16,13,10,7,6,1,0\}$.

Conjecture 2: For $A_{r, k}, N_{w^{1}}$ fails to be invertible iff one can find distinct primes $p_{i} \leq \min \{\bar{r}, k\}$ dividing $\bar{k}$ and nonnegative integers $a_{i}, b_{i}$ such that $\bar{r}=$ $\sum_{i} a_{i} p_{i}$ and $k=\sum_{i} b_{i} p_{i}$.

In other words, we conjecture that the condition of Thm. 6(3) is an 'iff'. Note that one way this condition will be satisfied is if $\operatorname{gcd}\{\bar{r}, k\} \neq 1$. The conditions in Thms. 6(4),(5) are strongest when we take $r<k$ (which without loss of generality we can). Also, the bound in $6(5)$ is best when the $p_{i}$ are labelled so that the largest are given indices near $n / 2$. In practice the most useful special case of Thms. 6(4),(5) is: If one can find an odd prime $p \leq \bar{r}$ for which $2 p$ divides $\bar{k}$ and $k \geq 3 p-1$, then $N_{w^{1}}$ will not be invertible. The analogue of Thm. 1(4) is also valid here, but is not very useful.

The answer to Question 2 for small $r$ and $k$ is indicated in the table. Computer checks were performed for $r \leq 9$ and all levels $k>r$ such that $\operatorname{dim} P_{+}^{r, k}<300,000$. The results were consistent with Conjecture 2. Conjectures 1 and 2 are the simplest guesses consistent with our results, but it would be nice to test them against additional numerical data.

Incidentally, conditions like ' $\ell \in \mathbb{Z}_{\geq}\left\{n_{1}, \ldots, n_{m}\right\}$ ' are only strong when $\ell$ is small. For example, given any coprime numbers $m$ and $n$, there are only $(m-$ 1) $(n-1) / 2$ positive integers $\ell$ which do not lie in $\mathbb{Z}_{\geq}\{m, n\}$ - the largest such $\ell$ 
is $m n-m-n$. So for fixed $\bar{r}$, we know Conjecture 2 will hold for all sufficiently large $k$.

\section{Extensions}

Because the fundamental weights are much simpler, the most interesting fusion-generators are the ones which consist only of fundamental weights: $\Gamma \subseteq$ $\left\{w^{1}, \ldots, w^{r}\right\}$. We can speak of fundamental-fusion-generators and fundamentalfusion-rank $\mathcal{F} \mathcal{R}_{r, k}$. All of the results in Sections 3 and 4 also apply directly to $\mathcal{F} \mathcal{R}_{r, k}$. By definition, $\mathcal{R}_{r, k} \leq \mathcal{F} \mathcal{R}_{r, k}$, and Conjecture 1 predicts that, for fixed $r, \mathcal{R}_{r, k}=\mathcal{F} \mathcal{R}_{r, k}$ for all sufficiently large $k$. Note however from the Table that $\mathcal{F R}_{8,5}=\mathcal{F R}_{4,9}=2$ while $\mathcal{R}_{8,5}=\mathcal{R}_{4,9}=1$.

Because of (2.8d), we can strengthen here the bound in Thm. 1(2). For example, if $\mathcal{F R}_{r, k}$ equals the bound given in Thm. 1(2), then so must $\mathcal{F} \mathcal{R}_{\bar{r} / d-1, k / d}$ for all divisors $d$ of $\operatorname{gcd}\{\bar{r}, k\}$.

One can also ask Question 2 for other weights, most importantly the other fundamental weights, and again $(2.8 \mathrm{~b})$ will be very useful. For example, we know $\chi_{w^{2}}$ will vanish at some $J^{5}$-fixed point of $A_{9,14}$, because $N_{w^{1}}$ is not invertible for $A_{4,7}$.

Of course Questions 1 and 2 can and should be asked of the fusion algebras for the other affine algebras, and similar arguments will apply. We have not investigated them, except to find some fusion-bases for $C_{2, k}$ and $G_{2, k}$ on the computer, and to get Thm. 7 below for $G_{2, k}$. Of course $\mathcal{R}_{k}\left(C_{2}^{(1)}\right)$ must equal 2 for any even $k$, and we find the rank is also 2 for all odd $k<26$ (the limit of our computer check), save $k=1,3$ and 9 . For $k=1$ and 9 , the only fusion-bases are $\left\{w^{1}\right\}$ and $\left\{2 w^{1}+6 w^{2}\right\}$, respectively. At $k=3$ there are four different fusion-bases: $\left\{2 w^{1}\right\},\left\{w^{2}\right\},\left\{2 w^{1}+w^{2}\right\}$, and $\left\{2 w^{2}\right\}$. A very tempting conjecture is that the rank $\mathcal{R}\left(C_{r, k}\right)$ equals 2 for all sufficiently large $k$ (and probably for all $k>9$ ). The situation for $G_{2, k}$ however is more surprising:

Theorem 7: (1) When the level $k$ is odd, $\left\{w^{2}\right\}$ is a fusion-basis for $G_{2, k}$. (2) $N_{w^{2}}$ fails to be invertible for $G_{2, k}$ iff either 4 or 30 divides $\bar{k}:=k+4$.

Proof The key here is to reduce the $G_{2, k}$ quantities to $A_{2, k+1}$ quantities, and use the fact that $\left\{w^{1}\right\}$ is a fusion-basis for $A_{2, k+1}$.

Using (1.1b) and the simple Lie subalgebra $A_{2} \subset G_{2}$, we find

$$
\chi_{w^{2}}(\mu)=\underline{\chi}_{\underline{w}^{1}}(\underline{\mu})+\underline{\chi}_{\underline{w}^{2}}(\underline{\mu})+1,
$$


where underlines denote $A_{2, k+1}$ quantities, and $\underline{\mu}=\mu_{1} \underline{w}^{1}+\left(\mu_{1}+\mu_{2}+1\right) \underline{w}^{2}$. So part (1) reduces to the following statement ${ }^{4}$ for $A_{2, k+1}$ : for any $\lambda, \mu \in P_{+}^{2, k+1}$ with $\lambda \neq C \lambda$ and $\mu \neq C \mu$ (only these nonselfconjugate weights correspond to $G_{2, k}$ ones), does the equality

$$
\begin{aligned}
& \cos \left(2 \pi \frac{\lambda_{1}+2 \lambda_{2}+3}{3 \bar{k}}\right)+\cos \left(2 \pi \frac{\lambda_{2}-\lambda_{1}}{3 \bar{k}}\right)+\cos \left(2 \pi \frac{2 \lambda_{1}+\lambda_{2}+3}{3 \bar{k}}\right) \\
& \quad=\cos \left(2 \pi \frac{\mu_{1}+2 \mu_{2}+3}{3 \bar{k}}\right)+\cos \left(2 \pi \frac{\mu_{2}-\mu_{1}}{3 \bar{k}}\right)+\cos \left(2 \pi \frac{2 \mu_{1}+\mu_{2}+3}{3 \bar{k}}\right)
\end{aligned}
$$

force either $\lambda=\mu$ or $\lambda=C \mu$ ?

Write $c_{1}, c_{2}, c_{3}$ for the three cosines on the left side of (5.2a), and write $c_{1}^{\prime}, c_{2}^{\prime}, c_{3}^{\prime}$ for those on the right. Then (5.2a) says $c_{1}+c_{2}+c_{3}=c_{1}^{\prime}+c_{2}^{\prime}+c_{3}^{\prime}$, and since $\left(2 \nu_{1}+\nu_{2}+3\right)+\left(\nu_{2}-\nu_{1}\right)=\nu_{1}+2 \nu_{2}+3$, we also get $c_{1}^{2}+c_{2}^{2}+c_{3}^{2}=1+2 c_{1} c_{2} c_{3}$ and $c_{1}^{\prime 2}+c_{2}^{\prime 2}+c_{3}^{\prime 2}=1+2 c_{1}^{\prime} c_{2}^{\prime} c_{3}^{\prime}$.

Hit both sides of (5.2a) with the Galois automorphism $\sigma_{2}$ (see Section 2). Since $\cos (2 x)=2 \cos ^{2}(x)-1$, we obtain

$$
c_{1}^{2}+c_{2}^{2}+c_{3}^{2}=c_{1}^{\prime 2}+c_{2}^{\prime 2}+c_{3}^{\prime 2} .
$$

Thus any symmetric polynomial in $c_{1}, c_{2}, c_{3}$ will equal the corresponding symmetric polynomial in $c_{1}^{\prime}, c_{2}^{\prime}, c_{3}^{\prime}$. In particular

$$
\begin{aligned}
& \left(\sin \left(2 \pi \frac{\lambda_{1}+2 \lambda_{2}+3}{3 \bar{k}}\right)-\sin \left(2 \pi \frac{\lambda_{2}-\lambda_{1}}{3 \bar{k}}\right)-\sin \left(2 \pi \frac{2 \lambda_{1}+\lambda_{2}+3}{3 \bar{k}}\right)\right)^{2} \\
& \quad=\left(\sin \left(2 \pi \frac{\mu_{1}+2 \mu_{2}+3}{3 \bar{k}}\right)-\sin \left(2 \pi \frac{\mu_{2}-\mu_{1}}{3 \bar{k}}\right)-\sin \left(2 \pi \frac{2 \mu_{1}+\mu_{2}+3}{3 \bar{k}}\right)\right)^{2} .
\end{aligned}
$$

In other words, we know from (5.2a) that the real parts of $\chi_{w^{1}}(\lambda)$ and $\chi_{w^{1}}(\mu)$ are equal, and from (5.2c) that their imaginary parts are also equal, up to a sign. Hence either $\lambda=\mu$ or $\lambda=C \mu$, and we have proven part (1).

For part (2), note that $\chi_{w^{2}}(\mu)=0$ is equivalent to (see (5.1))

$$
c_{1}+c_{2}+c_{3}=-\frac{1}{2},
$$

in the above notation. Consider first $k$ odd. Then hitting (5.3a) with the Galois automorphism $\sigma_{2}$ gives us $c_{1}^{2}+c_{2}^{2}+c_{3}^{2}=\frac{5}{4}$, and hence $c_{1} c_{2} c_{3}=\frac{1}{8}$. We can solve these equations, and we find $8 c_{i}^{3}+4 c_{i}^{2}-4 c_{i}-1=0$, i.e. $\left\{c_{1}, c_{2}, c_{3}\right\}=$

\footnotetext{
4 For the remainder of the proof of part (1), we will switch to $A_{2, k+1}$ notation.
} 
$\left\{\cos \left(2 \pi \frac{1}{7}\right), \cos \left(2 \pi \frac{2}{7}\right), \cos \left(2 \pi \frac{3}{7}\right)\right\}$. However, these cosines cannot be realised by a weight in $P_{+}^{k}\left(G_{2}^{(1)}\right)$.

Next, suppose $k \equiv 2(\bmod 4)$. We may assume (using $G_{2, k}$ notation) that exactly two of the arguments $\left\{3 \mu_{1}+2 \mu_{2}+5, \mu_{2}+1,3 \mu_{1}+\mu_{2}+4\right\}$ are odd, otherwise they would all be even and the argument would reduce to the $k$ odd one. Here we use the automorphism $\sigma_{3 \bar{k} / 2-2}$ and find (relabeling the $c_{i}$ if necessary) that $c_{3}^{2}-c_{1}^{2}-c_{2}^{2}=-\frac{3}{4}$. We can solve for $c_{i}$ as before, and we find that either $c_{3}=$ $\cos \left(2 \pi \frac{1}{5}\right)$ and $\left\{c_{1}, c_{2}\right\}=\left\{\cos \left(2 \pi \frac{7}{30}\right), \cos \left(2 \pi \frac{13}{30}\right)\right\}$, or $c_{3}=\cos \left(2 \pi \frac{2}{5}\right)$ and $\left\{c_{1}, c_{2}\right\}=$ $\left\{\cos \left(2 \pi \frac{1}{30}\right), \cos \left(2 \pi \frac{11}{30}\right)\right\}$. Either possibility requires 30 to divide $\bar{k}$, in order to be realised by a weight of $G_{2, k}$. When 30 divides $\bar{k}$, we do indeed get zeros: $\mu=(\bar{k} / 3-1, \bar{k} / 30-1,3 \bar{k} / 5-1) \in P_{+}^{k}\left(G_{2}^{(1)}\right)$ works.

Finally, suppose 4 divides $k$. Then $\mu=(k / 4, k / 4, k / 4) \in P_{+}^{k}\left(G_{2}^{(1)}\right)$ works.

(By $w^{2}$ here we mean the Weyl-dimension 7 fundamental weight of $G_{2}$, corresponding to the short simple root.) However, $\left\{w^{2}\right\}$ will not be a fusion-generator when $k>4$ is even. Our computer program tells us that for $k \leq 24$, the fusionrank is 1 except for $k=6,12,16$ and 20 (of course this implies it will also be 2 whenever $k+4$ is a multiple of 10,16 , or 24 ).

\section{Number fields associated with $S$}

By the field $K_{r, k}$ we mean the smallest field containing the rationals and all of the entries $S_{\lambda, \mu}$ of $S$. Similarly, by the field $L_{r, k}$ we mean the smallest field containing $\mathbb{Q}$ and all of the values $\chi_{\lambda}(\mu)$. Because of their role in the Galois symmetry (2.6), it is natural to try to identify these fields. This question was posed in [4], and related questions have been considered in e.g. [18,20]. Another reason the question is interesting is that, as we shall see, it has a simple answer! We will give this answer in Cor. 3 below, for the most important case: $A_{r, k}$.

The matrix $S$ for any nontwisted affine algebra $g$ is given in e.g. [16]. The expression for $S_{\lambda, \mu}$ consists of a sum $s(\lambda, \mu)$ over the Weyl group of $\bar{g}$, multiplied by a constant $c$. For $A_{r, k}, s(\lambda, \mu)$ manifestly lies in the field $\mathbb{Q}_{\bar{r} \bar{k}}$, and

$$
c=\frac{\mathrm{i}^{r(r+1) / 2}}{\bar{k}^{r / 2} \sqrt{r+1}} .
$$

Using Gauss sums, which express square-roots of integers as sums of roots of unity, it can be shown that the constant $c$ lies in either $\mathbb{Q}_{\bar{r}}$ if $r$ is even, or $\mathbb{Q}_{\bar{r} \bar{k}}$ if either 
$r \equiv 3(\bmod 4)$ or $k$ is even, or $\mathbb{Q}_{\bar{r} \bar{k}}[\sqrt{ \pm 2}]$ if both $k$ is odd and $k \bar{r} \equiv \pm 2(\bmod 8)$. Thus we know $L_{r, k}$ is always a subfield of $\mathbb{Q}_{\bar{r} \bar{k}}$, and $K_{r, k}$ is always a subfield of $\mathbb{Q}_{4 \bar{r} \bar{k}}$.

Write $[\lambda]$ for the orbit $\left\{J^{i} \lambda\right\}$ of $\lambda$ by the simple currents. We will find our fields by first computing some Galois orbits. This result should be of independent value.

Theorem 8: Consider any $k>2$ and $r \neq 1$.

(1) Choose any fundamental weight $w^{m}$ with $m \leq \min \{\bar{r}-2, k-2\}$, and any Galois automorphism $\sigma_{\ell}$. Then (with one exception) $\sigma_{\ell} w^{m} \in\left[w^{m}\right] \cup\left[C w^{m}\right]$ iff $\ell \equiv \pm 1(\bmod \bar{k}) ;$ for all other $\ell$ the quantum-dimension $S_{\sigma_{\ell} w^{m}, 0} / S_{0,0}$ of $\sigma_{\ell} w^{m}$ will be strictly greater than that of $w^{m}$. (The one exception is $w^{2}$ for $A_{3,4}$, where each $\sigma_{\ell}$ fixes $w^{2}$.)

(2) When $r \not \equiv 1(\bmod 4), \sigma_{\ell} w^{1}=w^{1} i f f \ell=1(\bmod \bar{r} \bar{k})$. When $r \equiv 1(\bmod 4)$ and $k$ is even, then $\sigma_{\ell} w^{1}=w^{1}$ iff $\ell=1(\bmod \bar{r} \bar{k} / 2)$.

Proof (1) Because of (2.1a), we may assume $m \leq \bar{r} / 2$. Assume first that $k \geq \bar{r}$. From the Weyl denominator formula, we compute

$$
\frac{S_{\sigma_{\ell} w^{m}, 0}}{S_{w^{m}, 0}}=\prod_{n=1}^{m} \frac{|\sin (\pi \ell n / \bar{k})|^{r-n}}{\sin (\pi n / \bar{k})^{r-n}} \prod_{n=m+1}^{\bar{r}-m} \frac{|\sin (\pi \ell n / \bar{k})|^{\bar{r}-n}}{\sin (\pi n / \bar{k})^{\bar{r}-n}} \prod_{n=\bar{r}+1-m}^{\bar{r}} \frac{|\sin (\pi \ell n / \bar{k})|^{\bar{r}+1-n}}{\sin (\pi n / \bar{k})^{\bar{r}+1-n}}
$$

where we drop the middle product if $m=\bar{r} / 2$. We want to know when $(6.2 \mathrm{a})$ equals 1. This is easy, for $k>r \geq 2$, since $\sin (\pi / \bar{k})<\sin (2 \pi / \bar{k})<\cdots<$ $\sin (\pi \bar{r} / \bar{k})$. Consider first $m<\bar{r} / 2$ : of all possible choices of integers $1 \leq n_{1}<$ $n_{2}<\cdots<n_{r+1} \leq \bar{k} / 2$, the minimum possible product of $r-1 \sin \left(\pi n_{1} / \bar{k}\right)$ 's, $r-2$ $\sin \left(\pi n_{2} / \bar{k}\right)^{\prime}$ 's, $\ldots, r-m \sin \left(\pi n_{m} / \bar{k}\right)$ 's, $r-m \sin \left(\pi n_{m+1} / \bar{k}\right)$ 's, $\ldots, m \sin \left(\pi n_{\bar{r}-m} / \bar{k}\right)$ 's, $m \sin \left(\pi n_{\bar{r}+1-m} / \bar{k}\right)$ 's, ..., and $1 \sin \left(\pi n_{\bar{r}} / \bar{k}\right)$, is the choice $n_{1}=1, n_{2}=2, \ldots$, $\left\{n_{m}, n_{m+1}\right\}=\{m, m+1\}, \ldots, n_{m+2}=m+2, \ldots,\left\{n_{\bar{r}-m}, n_{\bar{r}+1-m}\right\}=\{\bar{r}-m, \bar{r}+$ $1-m\}, \ldots, n_{m+1}=m+1$. This immediately forces $\ell \equiv \pm 1(\bmod \bar{k})($ for $m>1$, just look at the first term; when $m=1, \ell \equiv \pm 2$ is eliminated by seeing what happens to the second term).

If instead $m=\bar{r} / 2$, the exponents of $\sin (\pi n / \bar{k})$ in $(6.2 \mathrm{a})$ are no longer nonincreasing: near $n=m+1$ we get the subproduct

$\cdots \sin (\pi(m-1) / \bar{k})^{\bar{r}-m} \sin (\pi m / \bar{k})^{r-m} \sin (\pi(m+1) / \bar{k})^{\bar{r}-m} \sin (\pi(m+2) / \bar{k})^{r-m} \ldots$ 
For $m>2$, the proof that (6.2a) will always be greater than 1 for $\ell \not \equiv \pm 1$ $(\bmod \bar{k})$, follows from the simple observation that $\sin (\pi / \bar{k}) \sin (\pi(m+1) / \bar{k})<$ $\sin (2 \pi / \bar{k}) \sin (\pi m / \bar{k})$ : the least-harmful place to move ' 1 ' to is ' 2 ', and the best place to move ' $m+1$ ' to is ' $m$ ', and yet even that (forgetting the other terms, which will make matters worse) will increase the product. The remaining case $m=2$ corresponds to $\bar{r}=k=4$, i.e. to the given exception.

This completes the argument for $k \geq \bar{r}$. When $k<\bar{r}$, apply rank-level duality (2.3a): it is an exact symmetry of quantum-dimensions, and maps $J$-orbits to

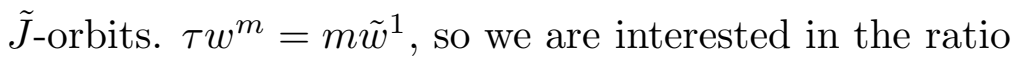

$$
\frac{\tilde{S}_{\sigma_{\ell} m \tilde{w}^{1}, 0}}{\tilde{S}_{m \tilde{w}^{1}, 0}}=\prod_{n=1}^{k-2} \frac{|\sin (\pi \ell n / \bar{k})|^{k-1-n}}{\sin (\pi n / \bar{k})^{k-1-n}} \prod_{n=1}^{k-1} \frac{|\sin (\pi \ell(n+m) / \bar{k})|}{\sin (\pi(n+m) / \bar{k})} .
$$

The rest of the argument is as before: again $m=\bar{r} / 2$ causes minor problems.

Now consider any $\ell=(-1)^{a}+b \bar{k}$. Applying $(2.6 \mathrm{~b})$ to the Cartan generators $\lambda \in\left\{w^{1}, \ldots, w^{r}\right\}$ and using $(2.4 \mathrm{e})$, we find

$$
\sigma_{\ell} \mu=C^{a} J^{b t(\mu+\rho)} \mu
$$

whenever $\sigma_{\ell} \in \operatorname{Gal}\left(L_{r, k} / \mathbb{Q}\right)$. Applying $(6.2 \mathrm{~b})$ to $\mu=w^{1}$ gives us part (2).

COROLlaRY 3: When both $k>2$ and $r \neq 1$, then $L_{r, k}=\mathbb{Q}_{\bar{r} \bar{k}}$ and

$$
K_{r, k}=\left\{\begin{array}{cc}
\mathbb{Q}_{\bar{r} \bar{k}} & \text { if either } r \not \equiv 1(\bmod 4) \text { or } k \text { is even } \\
\mathbb{Q}_{\bar{r} \bar{k}}[\sqrt{ \pm 2}] & \text { if } r \text { is odd and } \bar{r} k \equiv \pm 2(\bmod 8)
\end{array}\right.
$$

The proof of the Corollary is immediate from Thm. 8, by regarding Galois orbit sizes: when $r \not \equiv 1(\bmod 4)$, the Galois orbit of $w^{1}$ alone suffices, but when $r \equiv 1(\bmod 4)$ and $k$ is even, we have $\sigma_{1+\bar{r} \bar{k} / 2} w^{1}=w^{1}$, so also use $\sigma_{1+\bar{r} \bar{k} / 2} 0=$ $J^{\bar{r} / 2} 0 \neq 0$, which is obtained from $(6.2 \mathrm{~b})$. What we find in all cases is that for any $\ell \in(\mathbb{Z} / \bar{r} \bar{k} \mathbb{Z})^{\times}, \ell \neq 1$, either $\sigma_{\ell} w^{1} \neq w^{1}$ or $\sigma_{\ell} 0 \neq 0$. This tells us $L_{r, k}=\mathbb{Q}_{\bar{r} \bar{k}}$, and $K_{r, k}$ is then obtained by adjoining the constant $c$ shown above.

Similar statements to Thm. 8 can be found for other weights. For example, by rank-level duality the identical result to Thm. 8(1) holds for any $m w^{1}, 0 \leq$ $m \leq \min \{\bar{r}-2, k-2\}$, and we can expect similar results for other hooks. When $r \equiv 1(\bmod 4)$ and $k$ is odd, $\mathbb{Q}_{4 \bar{r} \bar{k}}$ is a degree 2 extension of $K_{r, k}$, which is in turn a degree 2 extension of $\mathbb{Q}_{\bar{r} \bar{k}}$. 
The results corresponding to Cor. 3 for $k=1,2$ or $r=1$ can be easily found, but are more complicated and hence less interesting. We include them here for completeness.

- $L_{r, 1}=\mathbb{Q}_{\bar{r}} . \quad K_{r, 1}$ will equal either $\mathbb{Q}_{\bar{r}}, \mathbb{Q}_{\bar{r}}[\mathrm{i}]$, or $\mathbb{Q}_{\bar{r}}[\sqrt{ \pm 2}]$, depending on whether or not $\bar{r} \equiv 0,1(\bmod 4)$, or $\bar{r} \equiv 3(\bmod 4)$, or $\bar{r} \equiv \pm 2(\bmod 8)$, respectively.

- $L_{1, k}=\mathbb{Q}[\cos (2 \pi / \bar{k})]$ if $k$ is odd, and $\mathbb{Q}[\cos (\pi / \bar{k})]$ if $k$ is even. $K_{1, k}$ will equal either $L_{1, k}$, or $L_{1, k}[\sqrt{2} \sin (2 \pi / \bar{k})]$, or $L_{1, k}[\sqrt{2}]$, depending on whether $k \equiv 0,2$, or $k \equiv 3$, or $k \equiv 1(\bmod 4)$, respectively.

- $L_{r, 2}=\mathbb{Q}_{\bar{r}}[\cos (2 \pi / \bar{k})]$ if $\bar{r}$ is odd, and $\mathbb{Q}_{\bar{r} \bar{k}}$ if $\bar{r}$ is even. $K_{r, 2}$ will equal $L_{r, 2}$, unless $\bar{r} \equiv 3(\bmod 4)$ when $K_{r, 2}=\mathbb{Q}_{\bar{r} \bar{k}}$. 


\section{Acknowledgements}

T.G. thanks A. Coste for showing him Questions 1 and 3, and C. Cummins

for discussions. M.W. thanks the High Energy Physics group of DAMTP for hospitality, and W. Eholzer for reading the manuscript.

\section{REFERENCES}

1. Aharony, O.: Generalized fusion potentials, Phys. Lett. B306 (1993), 276282.

2. Altschuler, D., Bauer, M., and Itzykson, C.: The branching rules of conformal embeddings, Commun. Math. Phys. 132 (1990), 349-364.

3. Bourbaki, N.: Groupes et Algèbres de Lie, Chapitres IV-VI, Hermann, Paris, 1968.

4. Buffenoir, E., Coste, A., Lascoux, J., Buhot, A., and Degiovanni, P.: Precise study of some number fields and Galois actions occurring in conformal field theory, Annales de l'I.H.P.: Phys. Théor. 63 (1995), 41-79.

5. Coste, A. and Gannon, T.: Remarks on Galois symmetry in rational conformal field theories, Phys. Lett. B323 (1994), 316-321.

6. Di Francesco, P. and Zuber, J.-B.: Fusion Potentials I, J. Phys. A26 (1993), 1441-1454.

7. Fuchs, J., Schellekens, B., and Schweigert, C.: From Dynkin diagram symmetries to fixed point structures, Commun. Math. Phys. 180 (1996), 39-97.

8. Fulton, W. and Harris, J.: Representation Theory: A First Course, SpringerVerlag, New York, 1991.

9. Gannon, T.: Symmetries of the Kac-Peterson modular matrices of affine algebras, Invent. Math. 122 (1995), 341-357.

10. Gannon, T.: Kac-Peterson, Perron-Frobenius, and the classification of conformal field theories, e-print q-alg/9510026, (1995).

11. Gannon, T., Ruelle, Ph., and Walton, M. A.: Automorphism modular invariants of current algebras, Commun. Math. Phys. 179 (1996), 121-156.

12. Georgieu, G. and Mathieu, O.: Catégorie de fusion pour les groupes de Chevalley, C. R. Acad. Sci. Paris 315 (1992), 659-662.

13. Gepner, D.: Fusion rings and geometry, Commun. Math. Phys. 141 (1991), 381-411.

14. Goodman, F. and Nakanishi, T.: Fusion algebras in integrable systems in two dimensions, Phys. Lett. B262 (1991), 259-264. 
15. Goodman, F. and Wenzl, H.: Littlewood-Richardson coefficients for Hecke algebras at roots of unity, Adv. Math. 82 (1990) 244-265.

16. Kac, V. and Peterson, D.: Infinite-dimensional Lie algebras, theta functions and modular forms, Adv. Math. 53 (1984), 125-264.

17. Kreuzer, M. and Schellekens, A. N.: Simple currents versus orbifolds with discrete torsion - a complete classification, Nucl. Phys. B411 (1994), 97-121.

18. Moody, R. V. and Patera, J.: Characters of elements of finite order in Lie groups, SIAM J. Alg. Disc. Meth. 5 (1984), 359-383.

19. Pasquier, V. and Saleur, H.: Common structures between finite systems and conformal field theories through quantum groups, Nucl. Phys. B330 (1990), 523-526.

20. Pianzola, A.: The arithmetic of the representation ring and elements of finite order in Lie groups, J. Algebra 108 (1987), 1-33.

21. Verlinde, E.: Fusion rules and modular transformations in 2D conformal field theory, Nucl. Phys. B300 (1988), 360-376.

22. Witten, E.: The Verlinde algebra and the cohomology of the Grassmannian, In: Geometry, Topology and Physics, Conf. Proc. and Lecture Notes in Geom. Topol. vol. VI (1995), 357-422. 